\title{
Rank-Based Estimation for GARCH Processes
}

\author{
Beth Andrews \\ Northwestern University
}

September 7, 2011

\begin{abstract}
We consider a rank-based technique for estimating GARCH model parameters, some of which are scale transformations of conventional GARCH parameters. The estimators are obtained by minimizing a rank-based residual dispersion function similar to the one given in Jaeckel (1972). They are useful for GARCH order selection and preliminary estimation. We give a limiting distribution for the rank estimators which holds when the true parameter vector is in the interior of its parameter space, and when some GARCH parameters are zero. The limiting theory is used to show that the rank estimators are robust, can have the same asymptotic efficiency as maximum likelihood estimators, and are relatively efficient compared to traditional Gaussian and Laplace quasi-maximum likelihood estimators. The behavior of the estimators for finite samples is studied via simulation, and we use rank estimation to fit a GARCH model to exchange rate log-returns.
\end{abstract}

The author is very grateful to editor Peter C.B. Phillips, co-editor Pentti Saikkonen, and two anonymous referees for their helpful comments. This work was supported in part by NSF Grant DMS0806104. Address correspondence to Beth Andrews, Department of Statistics, Northwestern University, Evanston, Illinois 60208, USA; e-mail: bandrews@northwestern.edu. 


\section{Introduction}

Observed time series processes frequently appear uncorrelated, yet exhibit volatility clustering. Volatility clustering is the tendency of observations relatively small in absolute value to be followed by other small observations, and the tendency of observations relatively large in absolute value to be followed by other large observations. Hence, these series appear uncorrelated, but dependent. Nonlinear models with time-dependent conditional variances, most notably generalized autoregressive conditionally heteroskedastic (GARCH) models, are often used to describe time series with these features. GARCH models were first developed for modeling inflation rates (Engle, 1982; Bollerslev, 1986), and have also appeared for analyzing the returns of exchange rates (Bollerslev, 1987; Engle and González-Rivera, 1991; Shephard, 1996) and stock prices (Bollerslev, 1987; Shephard, 1996; Fan and Yao, 2003). Applications for GARCH models are not limited to finance, however. Time series processes exhibiting GARCH-type behavior have also appeared, for example, in speech signals (Abramson and Cohen, 2008), daily and monthly mean temperatures (Campbell and Diebold, 2005; Romilly, 2005; Huang, Shiu, and Lin, 2008), wind speeds (Ewing, Kruse, and Thompson, 2008), and atmospheric carbon dioxide concentrations (Hoti, McAleer, and Chan, 2005).

In this paper, we consider a rank-based technique for estimating GARCH model parameters, some of which are scale transformations of conventional GARCH parameters. The rank $(\mathrm{R})$ estimators are obtained by minimizing the sum of mean-corrected model residuals weighted by a function of residual rank. They are similar to the R-estimators proposed by Jaeckel (1972) for estimating linear regression parameters, and can be used for GARCH order selection and preliminary estimation. As discussed in Jurečková and Sen (1996), R-estimators are, in general, robust and relatively efficient, and results in this paper indicate this is true in the case of GARCH estimation. The technique is robust because the R-estimators are $n^{1 / 2}$-consistent $(n$ represents sample size) under general conditions and, since the weight function can be chosen so that Restimators have the same asymptotic efficiency as maximum likelihood (ML) estimators, it is also relatively efficient. In addition, R-estimation dominates traditional techniques such as Gaussian and Laplace quasiML (QML) with respect to asymptotic efficiency. We, therefore, recommend that R-estimation be used for preliminary GARCH estimation and order selection when the noise distribution is unknown. Once a R-estimate has been found, corresponding model residuals can be used to identify an appropriate noise 
distribution, and then the conventional GARCH parameters can be estimated via ML.

Another rank-based technique for estimating the parameters of conditionally heteroskedastic processes is given in Mukherjee (2007). However, the class of models considered in Mukherjee (2007) includes ARCH but not GARCH models, and $n^{1 / 2}$-consistency for the $\mathrm{ARCH}$ parameter estimates is established only when the noise distribution has a finite fourth moment. While it is traditional to assume the noise distribution is Gaussian when fitting an ARCH/GARCH model to an observed series, many series appear to have noise distributions that are heavier-tailed than Gaussian (Bollerslev, 1987; Engle and González-Rivera, 1991; Shephard, 1996; Fan and Yao, 2003). Given the many applications for GARCH models, it is, therefore, important that robust statistical theory be developed. In this paper, we consider both ARCH and GARCH models and show that, when the true parameter vector lies in the interior of the parameter space, higher-order moment conditions are not required for the $n^{1 / 2}$-consistency of R-estimators of ARCH model parameters.

The R-estimators are introduced in Section 2 and, in Section 3.1, we show that as sample size $n \rightarrow \infty$, they converge in distribution to the minimizer of a random quadratic function on a convex space. This limiting result holds when the parameter vector lies in the interior of its parameter space, in which case it follows that the estimators are asymptotically normal, and also when some GARCH parameters are zero, and hence the parameter vector lies on the boundary of its parameter space. In Section 3.2, we show the limiting distribution for the R-estimators can be used for GARCH order selection and confidence interval estimation. Proofs of the lemmas used to establish the results of Section 3 are in the Appendix. The quality of the asymptotic approximations for finite samples is studied via simulation in Section 4.1, and we use R-estimation to fit a GARCH model to exchange rate log-returns in Section 4.2.

\section{Preliminaries}

A series $\left\{X_{t}\right\}_{t=-\infty}^{\infty}$ is a $\operatorname{GARCH}(p, q)$ process if

$$
X_{t}=\sigma_{t} Z_{t}
$$


where $\left\{Z_{t}\right\}$ is a sequence of independent and identically distributed (iid) random variables with $\mathrm{E}\left\{Z_{t}\right\}=0$ and $\operatorname{Var}\left\{Z_{t}\right\}=1,\left\{\sigma_{t}\right\}$ is a non-negative process satisfying

$$
\sigma_{t}^{2}=\alpha_{00}+\sum_{i=1}^{p} \alpha_{0 i} X_{t-i}^{2}+\sum_{j=1}^{q} \beta_{0 j} \sigma_{t-j}^{2},
$$

and $Z_{t}$ is independent of $\left\{X_{t-k}, k \geq 1\right\}$ for every $t$ (Bollerslev, 1986). The parameter $\alpha_{00}$ is positive, $\alpha_{0 i}, i \in$ $\{1, \ldots, p\}$, and $\beta_{0 j}, j \in\{1, \ldots, q\}$, are non-negative, and $\sigma_{t}^{2}$ represents $\operatorname{Var}\left\{X_{t} \mid X_{s}, s<t\right\}$. When $q=0$, $\left\{X_{t}\right\}$ is an $\operatorname{ARCH}(p)$ process (Engle, 1982). In the case of a $\operatorname{GARCH}(1,1)$ process, $\mathrm{E}\left\{\ln \left(\alpha_{01} Z_{t}^{2}+\beta_{01}\right)\right\}<0$ is necessary and sufficient for the stationarity and ergodicity of $\left\{X_{t}\right\}$ (Nelson, 1990) and, for $p \geq 2$ and $q \geq 2$, a stationary, ergodic solution to (2.1)-(2.2) exists if and only if the top Lyapunov exponent associated with the $(p+q-1) \times(p+q-1)$ matrices $\left\{\mathbf{A}_{0 t}\right\}$, where

$$
\mathbf{A}_{0 t}:=\left[\begin{array}{ccccccccc}
\alpha_{01} Z_{t}^{2}+\beta_{01} & \beta_{02} & \cdots & \beta_{0, q-1} & \beta_{0 q} & \alpha_{02} & \cdots & \alpha_{0, p-1} & \alpha_{0 p} \\
1 & 0 & \cdots & 0 & 0 & 0 & \cdots & 0 & 0 \\
0 & 1 & \cdots & 0 & 0 & 0 & \cdots & 0 & 0 \\
\vdots & \vdots & \ddots & \vdots & \vdots & \vdots & \ddots & \vdots & \vdots \\
0 & 0 & \cdots & 1 & 0 & 0 & \cdots & 0 & 0 \\
Z_{t}^{2} & 0 & \cdots & 0 & 0 & 0 & \cdots & 0 & 0 \\
0 & 0 & \cdots & 0 & 0 & 1 & \cdots & 0 & 0 \\
\vdots & \vdots & \ddots & \vdots & \vdots & \vdots & \ddots & \vdots & \vdots \\
0 & 0 & \cdots & 0 & 0 & 0 & \cdots & 1 & 0
\end{array}\right],
$$

is negative (Bougerol and Picard, 1992). That is, $\left\{X_{t}\right\}$ is stationary and ergodic if and only if $\gamma_{L}:=$ $\inf _{1 \leq t<\infty}\left[(t+1)^{-1} \mathrm{E}\left\{\ln \left\|\mathbf{A}_{00} \cdots \mathbf{A}_{0 t}\right\|_{o p}\right\}\right]<0$, where, for a matrix $\mathbf{A},\|\mathbf{A}\|_{o p}$ represents the matrix operator norm $\sup _{\mathbf{x} \neq \mathbf{0}}(\|\mathbf{A x}\| /\|\mathbf{x}\|)$, and $\|\cdot\|$ is the Euclidean norm. Note that $\gamma_{L}$ can also be used to assess the stationarity/ergodicity of a GARCH process for which $p<2$ or $q<2$ by setting $\alpha_{0 i}=0$ for $p<i \leq 2$ and $\beta_{0 j}=0$ for $q<j \leq 2$. Additionally, if $\sum_{i=1}^{p} \alpha_{0 i}+\sum_{j=1}^{q} \beta_{0 j}<1$, it can be shown that the process $\left\{X_{t}\right\}$ is not only stationary and ergodic, but also has finite variance $\alpha_{00} /\left(1-\sum_{i=1}^{p} \alpha_{0 i}-\sum_{j=1}^{q} \beta_{0 j}\right)$ (Bollerslev, 1986). We assume throughout that

A1. $\gamma_{L}<0$, 
so that $\left\{X_{t}\right\}$ is stationary and ergodic, but we do not assume the variance of $\left\{X_{t}\right\}$ is necessarily finite. Following Straumann (2005, page 76 ), so that the GARCH parameter values $\alpha_{00}, \alpha_{01}, \ldots, \alpha_{0 p}, \beta_{01}, \ldots, \beta_{0 q}$ are unique, we also assume

A2. there exists at least one $i>0$ for which $\alpha_{0 i}>0, \alpha_{0 p}+\beta_{0 q} \neq 0$, and the polynomials $\alpha_{0}(x):=\sum_{i=1}^{p} \alpha_{0 i} x^{i}$ and $\beta_{0}(x):=1-\sum_{j=1}^{q} \beta_{0 j} x^{j}$ have no common roots.

If we assume

A3. $P\left(Z_{t} \neq 0\right)=1$,

it follows from (2.1)-(2.2) that $\mathrm{P}\left(\cap_{t=-\infty}^{\infty}\left\{X_{t}^{2}>0\right\}\right)=1$. Therefore, we have $\ln \left(X_{t}^{2}\right)=\ln \left(\sigma_{t}^{2} / \alpha_{00}\right)+\ln \left(\alpha_{00} Z_{t}^{2}\right)$ or $\ln \left(\alpha_{00} Z_{t}^{2}\right)=\ln \left(X_{t}^{2}\right)-\ln \left(\sigma_{t}^{2} / \alpha_{00}\right)$, with $\sigma_{t}^{2} / \alpha_{00}=1+\sum_{i=1}^{p}\left(\alpha_{0 i} / \alpha_{00}\right) X_{t-i}^{2}+\sum_{j=1}^{q} \beta_{0 j}\left(\sigma_{t-j}^{2} / \alpha_{00}\right)$, and, for arbitrary values $\alpha_{0}, \alpha_{1}, \ldots, \alpha_{p}, \beta_{1}, \ldots, \beta_{q}$, we define

$$
\epsilon_{t}(\boldsymbol{\theta})=\ln \left(X_{t}^{2}\right)-\ln \left(\tilde{\sigma}_{t}^{2}(\boldsymbol{\theta})\right), \quad t=1, \ldots, n,
$$

with

$$
\tilde{\sigma}_{t}^{2}(\boldsymbol{\theta}):= \begin{cases}1, & t=\min \{1, p-q+1\}, \ldots, p, \\ 1+\sum_{i=1}^{p} \theta_{i} X_{t-i}^{2}+\sum_{j=1}^{q} \theta_{p+j} \tilde{\sigma}_{t-j}^{2}(\boldsymbol{\theta}), & t=p+1, \ldots, n,\end{cases}
$$

and $\boldsymbol{\theta}:=\left(\alpha_{1} / \alpha_{0}, \ldots, \alpha_{p} / \alpha_{0}, \beta_{1}, \ldots, \beta_{q}\right)^{\prime}$. Let $\boldsymbol{\theta}_{0}=\left(\alpha_{01} / \alpha_{00}, \ldots, \alpha_{0 p} / \alpha_{00}, \beta_{01}, \ldots, \beta_{0 q}\right)^{\prime}$ and $\epsilon_{t}=\ln \left(\alpha_{00} Z_{t}^{2}\right)$, and note that $\left\{\epsilon_{t}\left(\boldsymbol{\theta}_{0}\right)\right\}_{t=p+1}^{n}$ closely approximates $\left\{\epsilon_{t}\right\}_{t=p+1}^{n}$; the error is due to the initialization with ones in (2.4). Since A1 implies $\sum_{j=1}^{q} \beta_{0 j}<1$ (Bougerol and Picard, 1992, Corollary 2.3), $\boldsymbol{\theta}_{0}$ is in the parameter space $\boldsymbol{\Theta}:=[0, \infty)^{p} \times\left\{\left(x_{1}, \ldots, x_{q}\right)^{\prime} \in[0,1)^{q}: x_{1}+\cdots+x_{q}<1\right\}$. Now suppose

A4. $\lambda$ is a nonconstant and nondecreasing function from $(0,1)$ to $\mathbb{R}$.

For $\boldsymbol{\theta} \in \boldsymbol{\Theta}$, we introduce the R-function

$$
D_{n}(\boldsymbol{\theta})=\sum_{t=p+1}^{n} \lambda\left(\frac{R_{t}(\boldsymbol{\theta})}{n-p+1}\right)\left[\epsilon_{t}(\boldsymbol{\theta})-\overline{\epsilon(\boldsymbol{\theta})}\right],
$$

where $\left\{R_{t}(\boldsymbol{\theta})\right\}_{t=p+1}^{n}$ contains the ranks of the residuals $\left\{\epsilon_{t}(\boldsymbol{\theta})\right\}_{t=p+1}^{n}$ and $\overline{\epsilon(\boldsymbol{\theta})}:=(n-p)^{-1} \sum_{t=p+1}^{n} \epsilon_{t}(\boldsymbol{\theta})$. $D_{n}$ is similar to the R-function introduced in Jaeckel (1972) for estimating linear regression parameters. We, however, consider a weighted sum of the mean-corrected $\left\{\epsilon_{t}(\boldsymbol{\theta})\right\}_{t=p+1}^{n}$, instead of a weighted sum of the 
residuals (as in Jaeckel, 1972), to avoid assuming $\sum_{t=p+1}^{n} \lambda((t-p) /(n-p+1))=0$, which is required in Jaeckel (1972). Note that, if $\left\{\epsilon_{(t)}(\boldsymbol{\theta})\right\}_{t=p+1}^{n}$ is the series $\left\{\epsilon_{t}(\boldsymbol{\theta})\right\}_{t=p+1}^{n}$ ordered from smallest to largest, (2.5) can also be written as $D_{n}(\boldsymbol{\theta})=\sum_{t=p+1}^{n} \lambda((t-p) /(n-p+1))\left[\epsilon_{(t)}(\boldsymbol{\theta})-\overline{\epsilon(\boldsymbol{\theta})}\right]$ and, if $\bar{\lambda}:=(n-p)^{-1} \sum_{t=p+1}^{n} \lambda((t-$ $p) /(n-p+1)), D_{n}(\boldsymbol{\theta})=\sum_{t=p+1}^{n}[\lambda((t-p) /(n-p+1))-\bar{\lambda}]\left[\epsilon_{(t)}(\boldsymbol{\theta})-\overline{\epsilon(\boldsymbol{\theta})}\right]$. Because it tends to be near zero when the elements of $\left\{\epsilon_{t}(\boldsymbol{\theta})\right\}_{t=p+1}^{n}$ are similar and gets larger as the values of $\left\{\left|\epsilon_{(t)}(\boldsymbol{\theta})-\overline{\epsilon(\boldsymbol{\theta})}\right|\right\}_{t=p+1}^{n}$ increase, $D_{n}$ is a measure of the dispersion of the residuals $\left\{\epsilon_{t}(\boldsymbol{\theta})\right\}_{t=p+1}^{n}$. Given a realization of length $n$ from (2.1), $\left\{X_{t}\right\}_{t=1}^{n}$, we plan to estimate $\boldsymbol{\theta}_{0}$ by minimizing $D_{n}$. Our motivation for using the residuals $\left\{\epsilon_{t}(\boldsymbol{\theta})\right\}_{t=p+1}^{n}$ is that, given an appropriately chosen loss function, M-estimation with respect to $\left\{\epsilon_{t}(\boldsymbol{\theta})\right\}_{t=p+1}^{n}$ can be equivalent to ML or QML estimation for GARCH model parameters (Muler and Yohai, 2008). Additionally, it is not possible to estimate $\boldsymbol{\theta}_{0}$ by minimizing the dispersion of the residuals $\left\{X_{t} / \sqrt{\tilde{\sigma}_{t}^{2}(\boldsymbol{\theta})}\right\}_{t=p+1}^{n}$, since the values of $\left\{X_{t} / \sqrt{\tilde{\sigma}_{t}^{2}(\boldsymbol{\theta})}\right\}_{t=p+1}^{n}$ become more clustered about zero as the elements of $\boldsymbol{\theta}$ increase. By Theorem 2.1, $D_{n}$ is a non-negative, continuous function on $\Theta$. Choices for the weight function $\lambda$ are discussed in Section 3.1.

Theorem 2.1. Assume A1-A4 hold. If, for $\boldsymbol{\theta} \in \mathbf{\Theta}$,

$$
\left\{P_{1}(\boldsymbol{\theta}), \ldots, P_{(n-p) !}(\boldsymbol{\theta})\right\}=\left\{\left\{\epsilon_{1, p+1}(\boldsymbol{\theta}), \ldots, \epsilon_{1, n}(\boldsymbol{\theta})\right\}, \ldots,\left\{\epsilon_{(n-p) !, p+1}(\boldsymbol{\theta}), \ldots, \epsilon_{(n-p) !, n}(\boldsymbol{\theta})\right\}\right\}
$$

contains the $(n-p)$ ! permutations of the sequence $\left\{\epsilon_{t}(\boldsymbol{\theta})\right\}_{t=p+1}^{n}$ (so, for $j \in\{1, \ldots,(n-p) !\}, t \in\{p+1, \ldots, n\}$, $\epsilon_{j, t}(\boldsymbol{\theta})$ represents the $(t-p)$ th element of permutation $\left.P_{j}(\boldsymbol{\theta})\right)$, then

$$
D_{n}(\boldsymbol{\theta})=\sup _{j \in\{1, \ldots,(n-p) !\}} \sum_{t=p+1}^{n} \lambda\left(\frac{t-p}{n-p+1}\right)\left[\epsilon_{j, t}(\boldsymbol{\theta})-\overline{\epsilon(\boldsymbol{\theta})}\right] .
$$

In addition, $D_{n}$ is a non-negative, continuous function on $\Theta$.

Proof. Recall that $D_{n}(\boldsymbol{\theta})=\sum_{t=p+1}^{n}[\lambda((t-p) /(n-p+1))-\bar{\lambda}]\left[\epsilon_{(t)}(\boldsymbol{\theta})-\overline{\epsilon(\boldsymbol{\theta})}\right]$, and let $a_{n}(t)=\lambda((t-p) /(n-$ $p+1))-\bar{\lambda}$ and $z_{t}(\boldsymbol{\theta})=\epsilon_{t}(\boldsymbol{\theta})-\overline{\epsilon(\boldsymbol{\theta})}$. The results of this theorem follow from the proof of Theorem 1 in Jaeckel (1972), where properties are given for $\sum_{t=p+1}^{n} a_{n}(t) z_{(t)}(\boldsymbol{\theta})$.

We are, therefore, estimating $\boldsymbol{\theta}_{0}=\left(\alpha_{01} / \alpha_{00}, \ldots, \alpha_{0 p} / \alpha_{00}, \beta_{01}, \ldots, \beta_{0 q}\right)^{\prime}$ by minimizing the rank-based residual dispersion function (2.5). Other robust GARCH estimation techniques considered in the literature, M-estimation (Mukherjee, 2008; Muler and Yohai, 2008), least absolute deviations (Peng and Yao, 2003), and QML corresponding to noise distributions other than Gaussian (Berkes and Horváth, 2004), are also not 
used to directly estimate $\boldsymbol{\eta}_{0}:=\left(\alpha_{00}, \alpha_{01}, \ldots, \alpha_{0 p}, \beta_{01}, \ldots, \beta_{0 q}\right)^{\prime}$. Those methods are used instead to estimate $\left(\alpha_{00} / c, \alpha_{01} / c, \ldots, \alpha_{0 p} / c, \beta_{01}, \ldots, \beta_{0 q}\right)^{\prime}$, where $c>0$ is unknown when the noise distribution is unknown. Gaussian QML (Berkes, Horváth, and Kokoszka, 2003; Francq and Zakoïan, 2004) and ML (Berkes and Horváth, 2004) can be used to directly estimate $\boldsymbol{\eta}_{0}$ but, because Gaussian QMLEs have a rate of convergence slower than $n^{1 / 2}$ when $\mathrm{E}\left\{Z_{t}^{4}\right\}=\infty$ (Hall and Yao, 2003) and the noise distribution is unknown in practice, they are not robust techniques. Therefore, for estimating $\boldsymbol{\eta}_{0}$, we recommend that R-estimation be used as a preliminary technique. Once a R-estimate $\hat{\boldsymbol{\theta}}_{R}$ of $\boldsymbol{\theta}_{0}$ has been found, an appropriate noise distribution can be identified from the residuals $\left\{X_{t} / \sqrt{\tilde{\sigma}_{t}^{2}\left(\hat{\boldsymbol{\theta}}_{R}\right)}\right\}$, which resemble $\left\{\sqrt{\alpha_{00}} Z_{t}\right\}$ when $\hat{\boldsymbol{\theta}}_{R}$ is near $\boldsymbol{\theta}_{0}$, and $\boldsymbol{\eta}_{0}$ can be estimated via ML.

\section{$3 \quad$ Asymptotic Results}

\subsection{Limiting Distribution for R-Estimators}

Let $f$ and $F$ denote the density and distribution functions for $\ln \left(Z_{t}^{2}\right)$. In order to obtain the limiting distribution for R-estimators of $\boldsymbol{\theta}_{0}$, we make the following additional assumptions:

A5. $F$ is strictly increasing and differentiable on $\mathbb{R}$.

A6. $f$ is uniformly continuous on $\mathbb{R}$.

A7. The weight function $\lambda$ is bounded and left-continuous on $(0,1)$.

We also consider the following conditions:

A8. $\theta_{0 i}>0$ for all $i \in\{1, \ldots, p+q\}$.

A9. The set $\left\{j: \beta_{0 j}>0\right\} \neq \emptyset$, and $\prod_{i=1}^{j_{0}} \alpha_{0 i}>0$ for $j_{0}:=\min \left\{j: \beta_{0 j}>0\right\}$.

A10. $E X_{t}^{4}<\infty$.

Finally, for values of $\boldsymbol{\theta}=\left(\theta_{1}, \ldots, \theta_{p+q}\right)^{\prime} \in \boldsymbol{\Theta}$, we define the series $\left\{\epsilon_{t}^{*}(\boldsymbol{\theta})\right\}$ so that, for all $t, \epsilon_{t}^{*}(\boldsymbol{\theta})=$ $\ln \left(X_{t}^{2}\right)-\ln \left(\breve{\sigma}_{t}^{2}(\boldsymbol{\theta})\right)$ with

$$
\breve{\sigma}_{t}^{2}(\boldsymbol{\theta})=1+\sum_{i=1}^{p} \theta_{i} X_{t-i}^{2}+\sum_{j=1}^{q} \theta_{p+j} \breve{\sigma}_{t-j}^{2}(\boldsymbol{\theta}) .
$$


The sequence $\left\{\epsilon_{t}^{*}(\boldsymbol{\theta})\right\}_{t=p+1}^{n}$ is a stationary (stationarity follows from Berkes, Horváth, and Kokoszka, 2003, Section 2) approximation of the residuals $\left\{\epsilon_{t}(\boldsymbol{\theta})\right\}_{t=p+1}^{n}$. Note that $\left\{\breve{\sigma}_{t}^{2}\left(\boldsymbol{\theta}_{0}\right)\right\}=\left\{\sigma_{t}^{2} / \alpha_{00}\right\}$ and $\left\{\epsilon_{t}^{*}\left(\boldsymbol{\theta}_{0}\right)\right\}=$ $\left\{\epsilon_{t}\right\}$, with $\left\{\sigma_{t}^{2}\right\}$ defined in $(2.2)$ and $\epsilon_{t}=\ln \left(\alpha_{00} Z_{t}^{2}\right)$. Since $\sum_{j=1}^{q} \theta_{p+j}<1$ for $\boldsymbol{\theta}=\left(\theta_{1}, \ldots, \theta_{p+q}\right)^{\prime} \in \boldsymbol{\Theta}$, $1 /\left(1-\theta_{p+1} x-\cdots-\theta_{p+q} x^{q}\right)$ has a Laurent series expansion of the form $\sum_{j=0}^{\infty} c_{j}(\boldsymbol{\theta}) x^{j}$, where the coefficients $\left\{c_{j}(\boldsymbol{\theta})\right\}_{j=0}^{\infty}$ are geometrically decaying (Berkes, Horváth, and Kokoszka, 2003, Section 2). Because

$$
\frac{\partial \breve{\sigma}_{t}^{2}(\boldsymbol{\theta})}{\partial \theta_{i}}= \begin{cases}X_{t-i}^{2}+\sum_{j=1}^{q} \theta_{p+j}\left(\partial \breve{\sigma}_{t-j}^{2}(\boldsymbol{\theta}) / \partial \theta_{i}\right), & i \in\{1, \ldots, p\}, \\ \breve{\sigma}_{t+p-i}^{2}(\boldsymbol{\theta})+\sum_{j=1}^{q} \theta_{p+j}\left(\partial \breve{\sigma}_{t-j}^{2}(\boldsymbol{\theta}) / \partial \theta_{i}\right), & i \in\{p+1, \ldots, p+q\},\end{cases}
$$

it follows that the first partial derivatives of $\epsilon_{t}^{*}(\boldsymbol{\theta})$ are given by

$$
\frac{\partial \epsilon_{t}^{*}(\boldsymbol{\theta})}{\partial \theta_{i}}=\frac{-1}{\breve{\sigma}_{t}^{2}(\boldsymbol{\theta})} \frac{\partial \breve{\sigma}_{t}^{2}(\boldsymbol{\theta})}{\partial \theta_{i}}= \begin{cases}-\left(\sum_{j=0}^{\infty} c_{j}(\boldsymbol{\theta}) X_{t-i-j}^{2}\right) / \breve{\sigma}_{t}^{2}(\boldsymbol{\theta}), & i \in\{1, \ldots, p\}, \\ -\left(\sum_{j=0}^{\infty} c_{j}(\boldsymbol{\theta}) \breve{\sigma}_{t+p-i-j}^{2}(\boldsymbol{\theta})\right) / \breve{\sigma}_{t}^{2}(\boldsymbol{\theta}), & i \in\{p+1, \ldots, p+q\} .\end{cases}
$$

When $\boldsymbol{\theta}=\boldsymbol{\theta}_{0}$,

$$
\frac{\partial \epsilon_{t}^{*}\left(\boldsymbol{\theta}_{0}\right)}{\partial \theta_{i}}= \begin{cases}-\alpha_{00}\left(\sum_{j=0}^{\infty} c_{j}\left(\boldsymbol{\theta}_{0}\right) X_{t-i-j}^{2}\right) / \sigma_{t}^{2}, & i \in\{1, \ldots, p\}, \\ -\left(\sum_{j=0}^{\infty} c_{j}\left(\boldsymbol{\theta}_{0}\right) \sigma_{t+p-i-j}^{2}\right) / \sigma_{t}^{2}, & i \in\{p+1, \ldots, p+q\}\end{cases}
$$

(these are right derivatives when $\left.\theta_{0 i}=0\right)$, with $\sum_{j=0}^{\infty} c_{j}\left(\boldsymbol{\theta}_{0}\right) x^{j}=1 /\left(1-\beta_{01} x-\cdots-\beta_{0 q} x^{q}\right)$. We are now able to give the limiting distribution for R-estimators of $\boldsymbol{\theta}_{0}$.

Theorem 3.1. If $A 1-A 7$ and either $A 8, A 9$ or $A 10$ hold, then there exists a sequence of minimizers $\hat{\boldsymbol{\theta}}_{R} \in \mathbf{\Theta}$ of $D_{n}(\cdot)$ in (2.5) such that

$$
n^{1 / 2}\left(\hat{\boldsymbol{\theta}}_{R}-\boldsymbol{\theta}_{0}\right) \stackrel{d}{\rightarrow} \boldsymbol{\xi}:=\operatorname{argmin}_{\mathbf{u} \in \Lambda}(\mathbf{u}-\mathbf{Y})^{\prime} \tilde{K} \boldsymbol{\Gamma}(\mathbf{u}-\mathbf{Y})
$$

where $\Lambda=\Lambda_{1} \times \cdots \times \Lambda_{p+q}$, with $\Lambda_{i}=\mathbb{R}$ if $\theta_{0 i}>0$ and $\Lambda_{i}=[0, \infty)$ if $\theta_{0 i}=0, \mathbf{Y} \sim N(\mathbf{0}, \boldsymbol{\Sigma}), \boldsymbol{\Sigma}:=\tilde{J} \tilde{K}^{-2} \boldsymbol{\Gamma}^{-1}$, $\tilde{J}:=\int_{0}^{1} \lambda^{2}(x) d x-\left(\int_{0}^{1} \lambda(x) d x\right)^{2}, \tilde{K}:=\int_{-\infty}^{\infty} f(x) d \lambda(F(x))$, and

$$
\boldsymbol{\Gamma}:=E\left\{\left[\frac{\partial \epsilon_{t}^{*}\left(\boldsymbol{\theta}_{0}\right)}{\partial \boldsymbol{\theta}}-E\left\{\frac{\partial \epsilon_{t}^{*}\left(\boldsymbol{\theta}_{0}\right)}{\partial \boldsymbol{\theta}}\right\}\right]\left[\frac{\partial \epsilon_{t}^{*}\left(\boldsymbol{\theta}_{0}\right)}{\partial \boldsymbol{\theta}}-E\left\{\frac{\partial \epsilon_{t}^{*}\left(\boldsymbol{\theta}_{0}\right)}{\partial \boldsymbol{\theta}}\right\}\right]^{\prime}\right\} .
$$

The limiting random vector $\boldsymbol{\xi}$ is a unique, finite value almost surely.

Proof. By Lemma 5.4 in the Appendix, $S_{n}(\mathbf{u})=D_{n}\left(\boldsymbol{\theta}_{0}+n^{-1 / 2} \mathbf{u}\right)-D_{n}\left(\boldsymbol{\theta}_{0}\right) \stackrel{d}{\rightarrow} S(\mathbf{u})=\mathbf{u}^{\prime} \mathbf{N}+\tilde{K} \mathbf{u}^{\prime} \mathbf{\Gamma u} / 2$ on $C(\Lambda)$, where $\mathbf{N} \sim \mathrm{N}(\mathbf{0}, \tilde{J} \boldsymbol{\Gamma})$ and $C(\Lambda)$ is the space of continuous functions on $\Lambda$ where convergence is 
equivalent to uniform convergence on every compact subset. The derivatives $\partial \epsilon_{t}^{*}\left(\boldsymbol{\theta}_{0}\right) / \partial \theta_{i}, i \in\{1, \ldots, p+q\}$, have finite second moments (in the case of A8 this follows from the proof of Theorem 2.2(i) in Francq and Zakoïan, 2004, and in the case of A9 or A10 this follows from the proof of Lemma 8 in Francq and Zakoïan, 2007) so the matrix $\boldsymbol{\Gamma}$ exists, and, following the proof of Theorem 2.2(ii) in Francq and Zakoïan (2004), $\boldsymbol{\Gamma}$ is positive definite. Consequently, since $\tilde{K}>0$ and $\Lambda$ is a convex space, $S(\mathbf{u})$ has a unique minimum on $\Lambda$ almost surely. Because $n^{1 / 2}\left(\hat{\boldsymbol{\theta}}_{R}-\boldsymbol{\theta}_{0}\right)$ minimizes $S_{n}(\mathbf{u})$, it follows from the proof of Lemma 2.2 and Remark 1 in Davis, Knight, and Liu (1992) that $n^{1 / 2}\left(\hat{\boldsymbol{\theta}}_{R}-\boldsymbol{\theta}_{0}\right) \stackrel{d}{\rightarrow} \operatorname{argmin}_{\mathbf{u} \in \Lambda} S(\mathbf{u})$. The result of this theorem holds since $\mathbf{N} \stackrel{d}{=}-\tilde{K} \mathbf{\Gamma Y}$, which implies that $\operatorname{argmin}_{\mathbf{u} \in \Lambda} S(\mathbf{u}) \stackrel{d}{=} \operatorname{argmin}_{\mathbf{u} \in \Lambda}\left(\mathbf{Y}^{\prime} \tilde{K} \mathbf{\Gamma} \mathbf{Y}-\right.$ $\left.2 \mathbf{u}^{\prime} \tilde{K} \mathbf{\Gamma} \mathbf{Y}+\mathbf{u}^{\prime} \tilde{K} \boldsymbol{\Gamma} \mathbf{u}\right)=\operatorname{argmin}_{\mathbf{u} \in \Lambda}(\mathbf{u}-\mathbf{Y})^{\prime} \tilde{K} \boldsymbol{\Gamma}(\mathbf{u}-\mathbf{Y})$.

If all parameter values are positive (ie., A8 holds), it follows from Theorem 3.1 that $\hat{\boldsymbol{\theta}}_{R}$ is asymptotically normal.

Corollary 3.1. If A1-A8 hold, then

$$
n^{1 / 2}\left(\hat{\boldsymbol{\theta}}_{R}-\boldsymbol{\theta}_{0}\right) \stackrel{d}{\rightarrow} \mathbf{Y} \sim N(\mathbf{0}, \boldsymbol{\Sigma})
$$

Proof. This result follows from (3.2), since $\tilde{K} \boldsymbol{\Gamma}$ is positive definite and, under $\mathrm{A} 8, \Lambda=\mathbb{R}^{p+q}$.

Remark 1: Assumptions A5 and A6 are mild. They hold, for example, if $Z_{t}$ has a Laplace, logistic, N(0,1), or rescaled Student's $t$ (rescaled to have unit variance) distribution.

Remark 2: As discussed in the proof of Theorem 3.1, given A8, A9 or A10, the derivatives $\partial \epsilon_{t}^{*}\left(\boldsymbol{\theta}_{0}\right) / \partial \theta_{i}$, $i \in\{1, \ldots, p+q\}$, have finite second moments. Since A8 is required for GARCH parameter estimates to be asymptotically unbiased and normal, it is a standard assumption in the literature. Conditions A9 and A10 are similar to assumptions introduced by Francq and Zakoïan (2007), who derived the limiting distribution for Gaussian QMLEs when some GARCH parameter values may be zero. In the absence of A8 and A9, $\mathrm{E} X_{t}^{6}<\infty$, instead of A10 $\left(\mathrm{E} X_{t}^{4}<\infty\right)$, is required for the $n^{1 / 2}$-consistency of the Gaussian QMLEs, however (Francq and Zakoïan, 2007). Note that $\alpha_{01}>0$ and $\beta_{01}>0$ are sufficient for A9. 
Remark 3: When a parameter vector may lie on a boundary of its parameter space, it is standard for estimators to converge in distribution to the minimizer of a random quadratic function on a convex space (D.W.K. Andrews, 1999). Francq and Zakoïan (2007) show this is true for Gaussian QMLEs of GARCH model parameters. The form of the limiting distribution for $\hat{\boldsymbol{\theta}}_{R}$ in (3.2) is, therefore, to be expected. Let the vector $\left(Y_{1}, \ldots, Y_{p+q}\right)^{\prime}$ contain the Gaussian random elements of $\mathbf{Y} \sim \mathrm{N}(\mathbf{0}, \boldsymbol{\Sigma})$. If only the $j$ th element of $\boldsymbol{\theta}_{0}, \theta_{0 j}$, equals zero and all other elements of $\boldsymbol{\theta}_{0}$ are positive, it follows from (3.2) that $n^{1 / 2}\left(\hat{\theta}_{j, R}-\theta_{0 j}\right)=$ $n^{1 / 2} \hat{\theta}_{j, R} \stackrel{d}{\rightarrow} Y_{j} I\left\{Y_{j} \geq 0\right\}\left(I\{\cdot\}\right.$ represents the indicator function), so $\hat{\theta}_{j, R}$ is asymptotically half-normal in this case.

Remark 4: If $f$, the density function for $\ln \left(Z_{t}^{2}\right)$, is almost everywhere differentiable on $\mathbb{R}$, using integration by parts, it can be shown that $\tilde{K}=-\int_{-\infty}^{\infty} f^{\prime}(x) \lambda(F(x)) d x=-\int_{0}^{1}\left[f^{\prime}\left(F^{-1}(x)\right) / f\left(F^{-1}(x)\right)\right] \lambda(x) d x$. In practice, these integrals can be easier to evaluate than $\int_{-\infty}^{\infty} f(x) d \lambda(F(x))$.

Remark 5: Let $f_{Z}$ represent the density function for the iid noise process $\left\{Z_{t}\right\}$ and, for $\boldsymbol{\eta}=\left(\eta_{0}, \eta_{1}, \ldots, \eta_{p+q}\right)^{\prime} \in(0, \infty) \times \boldsymbol{\Theta}$, let $\epsilon_{t}^{\dagger}(\boldsymbol{\eta})=\ln \left(X_{t}^{2}\right)-\ln \left(\sigma_{t}^{2}(\boldsymbol{\eta})\right)$ with $\sigma_{t}^{2}(\boldsymbol{\eta})=\eta_{0}+\sum_{i=1}^{p} \eta_{i} X_{t-i}^{2}+$ $\sum_{j=1}^{q} \eta_{p+j} \sigma_{t-j}^{2}(\boldsymbol{\eta}) \forall t$. From Berkes and Horváth (2004), under general conditions which include A8, ML estimators of $\boldsymbol{\eta}_{0}=\left(\alpha_{00}, \alpha_{01}, \ldots, \alpha_{0 p}, \beta_{01}, \ldots, \beta_{0 q}\right)^{\prime}$ are asymptotically normal with mean $\boldsymbol{\eta}_{0}$ and covariance matrix $4 n^{-1} \tau^{2} \mathbf{A}^{-1}$, where $\tau^{2}=\left(\mathrm{E}\left\{\left[1+Z_{t} f_{Z}^{\prime}\left(Z_{t}\right) / f_{Z}\left(Z_{t}\right)\right]^{2}\right\}\right)^{-1}$ and $\mathbf{A}=\mathrm{E}\left\{\left[\partial \epsilon_{t}^{\dagger}\left(\boldsymbol{\eta}_{0}\right) / \partial \boldsymbol{\eta}\right]\left[\partial \epsilon_{t}^{\dagger}\left(\boldsymbol{\eta}_{0}\right) / \partial \boldsymbol{\eta}\right]^{\prime}\right\}$. Using the delta method (see, for example, Rao, 1973, pages 387-388), the corresponding estimators of $\boldsymbol{\theta}_{0}$, $\hat{\boldsymbol{\theta}}_{M L}:=\left(\hat{\alpha}_{1, M L} / \hat{\alpha}_{0, M L}, \ldots, \hat{\alpha}_{p, M L} / \hat{\alpha}_{0, M L}, \hat{\beta}_{1, M L}, \ldots, \hat{\beta}_{q, M L}\right)^{\prime}$, are asymptotically Gaussian with mean $\boldsymbol{\theta}_{0}$ and covariance matrix $4 n^{-1} \tau^{2} \mathbf{B} \mathbf{A}^{-1} \mathbf{B}^{\prime}$, where $\mathbf{B}$ is the $(p+q) \times(p+q+1)$ Jacobian matrix

$$
\mathbf{B}=\left[\begin{array}{cccccccc}
-\alpha_{01} / \alpha_{00}^{2} & 1 / \alpha_{00} & 0 & \cdots & 0 & 0 & \cdots & 0 \\
-\alpha_{02} / \alpha_{00}^{2} & 0 & 1 / \alpha_{00} & \cdots & 0 & 0 & \cdots & 0 \\
\vdots & \vdots & \vdots & \ddots & \vdots & \vdots & \ddots & \vdots \\
-\alpha_{0 p} / \alpha_{00}^{2} & 0 & 0 & \cdots & 1 / \alpha_{00} & 0 & \cdots & 0 \\
0 & 0 & 0 & \cdots & 0 & 1 & \cdots & 0 \\
\vdots & \vdots & \vdots & \ddots & \vdots & \vdots & \ddots & \vdots \\
0 & 0 & 0 & \cdots & 0 & 0 & \cdots & 1
\end{array}\right] .
$$

For any $p$ and $q$, matrix algebra can be used to show that $\mathbf{B} \mathbf{A}^{-1} \mathbf{B}^{\prime}=\boldsymbol{\Gamma}^{-1}$ and, therefore, under assumptions 
A1-A8, the asymptotic relative efficiency (ARE) for R-estimation with respect to ML is $4 \tau^{2} \tilde{J}^{-1} \tilde{K}^{2}$.

If the weight function $\lambda$ is proportional to $-f^{\prime}\left(F^{-1}(x)\right) / f\left(F^{-1}(x)\right)$, then $\tilde{J}^{-1} \tilde{K}^{2}=$ $\mathrm{E}\left\{\left[f^{\prime}\left(\ln \left(Z_{t}^{2}\right)\right) / f\left(\ln \left(Z_{t}^{2}\right)\right)\right]^{2}\right\}$ (Jurečková and Sen, 1996, Section 3.4). In addition, since $f(x)=\sqrt{e^{x}} f_{Z}\left(\sqrt{e^{x}}\right)$ when the distribution for $Z_{t}$ is symmetric about zero, it can be shown that $f^{\prime}\left(\ln \left(Z_{t}^{2}\right)\right) / f\left(\ln \left(Z_{t}^{2}\right)\right)=[1+$ $\left.Z_{t} f_{Z}^{\prime}\left(Z_{t}\right) / f_{Z}\left(Z_{t}\right)\right] / 2$ in the symmetric case. Consequently, when the distribution for $Z_{t}$ is symmetric about zero and a weight function

$$
\lambda_{f}(x) \propto-f^{\prime}\left(F^{-1}(x)\right) / f\left(F^{-1}(x)\right)
$$

is used, $\tilde{J}^{-1} \tilde{K}^{2}=\mathrm{E}\left\{\left[1+Z_{t} f_{Z}^{\prime}\left(Z_{t}\right) / f_{Z}\left(Z_{t}\right)\right]^{2}\right\} / 4=\left(4 \tau^{2}\right)^{-1}$ and, if A1-A8 hold, R-estimation has the same asymptotic efficiency as ML.

Remark 6: When $Z_{t} \sim \mathrm{N}(0,1)$, the optimal weight function $\lambda_{f}(x) \propto\left[\Phi^{-1}((x+1) / 2)\right]^{2}-1$, where $\Phi$ represents the standard normal distribution function. Hence, $\tilde{J}^{-1} \tilde{K}^{2}=\left(4 \tau^{2}\right)^{-1}$ when $Z_{t} \sim \mathrm{N}(0,1)$ and $\lambda(x)=\left[\Phi^{-1}((x+1) / 2)\right]^{2}-1$. However, the function $\lambda_{N}(x):=\left[\Phi^{-1}((x+1) / 2)\right]^{2}-1$ does not satisfy assumption A7, since it is unbounded as $x \rightarrow 1$, so limiting results (3.2) and (3.3) do not necessarily hold for R-estimates obtained using $\lambda_{N}$. Bounded weight functions closely approximating $\lambda_{N}$ which do satisfy the assumptions can, however, be found. For example, let $\lambda_{m, N}(x)=\lambda_{N}(x) I\{0<x \leq 1-1 / m\}+$ $\lambda_{N}(1-1 / m) I\{x>1-1 / m\}$, with $m \geq 2$. This weight function satisfies assumptions A4 and A7 and, as $m \rightarrow \infty, \lambda_{m, N}$ converges pointwise to $\lambda_{N}$ on $(0,1)$. In addition, $\tilde{J}_{m}:=\int_{0}^{1} \lambda_{m, N}^{2}(x) d x-\left(\int_{0}^{1} \lambda_{m, N}(x) d x\right)^{2} \rightarrow$ $\int_{0}^{1} \lambda_{N}^{2}(x) d x-\left(\int_{0}^{1} \lambda_{N}(x) d x\right)^{2}$ and $\tilde{K}_{m}:=\int_{-\infty}^{\infty} f(x) d \lambda_{m, N}(F(x)) \rightarrow \int_{-\infty}^{\infty} f(x) d \lambda_{N}(F(x))$. Therefore, in the case of Gaussian noise, large $m \geq 2$ can be chosen so that R-estimation with weight function $\lambda_{m, N}$ has essentially the same asymptotic efficiency as ML.

Gaussian ML estimators of GARCH model parameters are also consistent when the noise distribution is non-Gaussian (Francq and Zakoïan, 2004), and when all parameter values are positive and $\mathrm{E}\left\{Z_{t}^{4}\right\}<\infty$, Gaussian QMLEs of $\boldsymbol{\eta}_{0}$ are asymptotically normal with mean $\boldsymbol{\eta}_{0}$ and covariance matrix $n^{-1}\left(\mathrm{E}\left\{Z_{t}^{4}\right\}-1\right) \mathbf{A}^{-1}$ (Berkes and Horváth, 2004; Francq and Zakoïan, 2004). It follows that the corresponding estimators of $\boldsymbol{\theta}_{0}$, $\hat{\boldsymbol{\theta}}_{G M L}:=\left(\hat{\alpha}_{1, G M L} / \hat{\alpha}_{0, G M L}, \ldots, \hat{\alpha}_{p, G M L} / \hat{\alpha}_{0, G M L}, \hat{\beta}_{1, G M L}, \ldots, \hat{\beta}_{q, G M L}\right)^{\prime}$, have a limiting normal distribution with mean $\boldsymbol{\theta}_{0}$ and covariance matrix $n^{-1}\left(\mathrm{E}\left\{Z_{t}^{4}\right\}-1\right) \mathbf{B} \mathbf{A}^{-1} \mathbf{B}^{\prime}=n^{-1}\left(\mathrm{E}\left\{Z_{t}^{4}\right\}-1\right) \boldsymbol{\Gamma}^{-1}$, so the ARE for Restimation with respect to Gaussian QML is $\left(\mathrm{E}\left\{Z_{t}^{4}\right\}-1\right) \tilde{J}^{-1} \tilde{K}^{2}$. The rate of convergence for Gaussian 


\begin{tabular}{|c|c|}
\hline Distribution for Noise $\left\{Z_{t}\right\}$ & ARE \\
\hline Laplace & 1.221 \\
logistic & 1.118 \\
$\mathrm{~N}(0,1)$ & 1.000 \\
$t_{3}$ & $\infty$ \\
$t_{5}$ & 2.321 \\
$t_{7}$ & 1.341 \\
$t_{9}$ & 1.167 \\
$t_{12}$ & 1.082 \\
$t_{15}$ & 1.049 \\
$t_{20}$ & 1.026 \\
$t_{30}$ & 1.011 \\
\hline
\end{tabular}

Table 3.1: AREs for R-estimation with $\lambda(x) \approx \lambda_{N}(x)$ with respect to Gaussian $Q M L$.

QMLEs is slower than $n^{1 / 2}$ when $\mathrm{E}\left\{Z_{t}^{4}\right\}=\infty$, however (Hall and Yao, 2003). Since $\mathrm{E}\left\{Z_{t}^{4}\right\}<\infty$ is not required for the $n^{1 / 2}$-consistency of $\hat{\boldsymbol{\theta}}_{R}$, R-estimation is more robust than traditional Gaussian QML.

In Table 3.1 we give the values of $\operatorname{ARE}\left(\mathrm{E}\left\{Z_{t}^{4}\right\}-1\right) \tilde{J}^{-1} \tilde{K}^{2}$ (rounded to the nearest three decimal places) when the weight function is $\lambda_{m, N}$ with $m$ large (ie., $\lambda(x) \approx \lambda_{N}(x)$ ) and the noise distribution is Laplace, logistic, $\mathrm{N}(0,1)$, and rescaled $t$ with various degrees of freedom. Note that, in the case of rescaled $t_{3}$ noise, we have an ARE of $\infty$, since in this case $\mathrm{E}\left\{Z_{t}^{4}\right\}=\infty$ and so Gaussian QMLEs have a rate of convergence slower than $n^{1 / 2}$. Since all AREs in Table 3.1 are greater than or equal to one, with equality only when the noise distribution is Gaussian, R-estimation is not only more robust than Gaussian QML, but also tends to be more efficient. In the case of R-estimation for linear model parameters, R-estimation with the weight function that is optimal for Gaussian noise is always at least as asymptotically efficient as Gaussian QML (Chernoff and Savage, 1958; Gastwirth and Wolff, 1968; see Hettmansperger and McKean, 1998, for discussion). As can be seen in Table 3.1, this is true in the case of GARCH parameter estimation for commonly used noise distributions, but it is possible for $\left(\mathrm{E}\left\{Z_{t}^{4}\right\}-1\right) \tilde{J}^{-1} \tilde{K}^{2}$ to be much less than one when $\lambda(x) \approx\left[\Phi^{-1}((x+1) / 2)\right]^{2}-1$, however (for example, when $\ln \left(Z_{t}^{2}\right) \sim \mathrm{N}\left(\mu=-0.125, \sigma^{2}=0.25\right.$ ), $\left.\left(\mathrm{E}\left\{Z_{t}^{4}\right\}-1\right) \tilde{J}^{-1} \tilde{K}^{2}=0.787\right)$.

Remark 7: R-estimation is also relatively efficient when compared to more robust GARCH estimation techniques. To demonstrate, in Table 3.2, we give AREs for R-estimation with weight function $\lambda_{t_{7}}(x):=$ $\left[7\left\{F_{t_{7}}^{-1}((x+1) / 2)\right\}^{2}-5\right] /\left[\left\{F_{t_{7}}^{-1}((x+1) / 2)\right\}^{2}+5\right]$, where $F_{t_{7}}$ represents the distribution function for rescaled $t_{7}$ noise, with respect to (A) Laplace QML (Berkes and Horváth, 2004; Mukherjee, 2008), (B) rescaled 


\begin{tabular}{|c|cccc|}
\hline & \multicolumn{4}{|c|}{ ARE } \\
Distribution for Noise $\left\{Z_{t}\right\}$ & $(\mathrm{A})$ & $(\mathrm{B})$ & $(\mathrm{C})$ & $(\mathrm{D})$ \\
\hline Laplace & 0.986 & 1.014 & 2.053 & 1.233 \\
logistic & 1.013 & 1.002 & 2.097 & 1.139 \\
$\mathrm{~N}(0,1)$ & 1.094 & 1.041 & 2.607 & 0.958 \\
$t_{3}$ & 1.411 & 1.052 & 1.551 & $\infty$ \\
$t_{5}$ & 1.058 & 1.005 & 1.867 & 2.489 \\
$t_{7}$ & 1.029 & 1.000 & 2.040 & 1.400 \\
$t_{9}$ & 1.028 & 1.002 & 2.147 & 1.198 \\
$t_{12}$ & 1.036 & 1.007 & 2.250 & 1.093 \\
$t_{15}$ & 1.043 & 1.011 & 2.315 & 1.050 \\
$t_{20}$ & 1.053 & 1.017 & 2.383 & 1.016 \\
$t_{30}$ & 1.064 & 1.024 & 2.454 & 0.991 \\
\hline
\end{tabular}

Table 3.2: AREs for R-estimation with weight function $\lambda_{t_{7}}$ with respect to (A) Laplace $Q M L$, (B) rescaled Student's $t_{7} Q M L,(C)$ least absolute deviations, and (D) Gaussian QML.

Student's $t_{7}$ QML (Muler and Yohai, 2008), and (C) the least absolute deviations estimation technique proposed by Peng and Yao (2003) involving log-transformed GARCH residuals. The weight function $\lambda_{t_{7}}$ is optimal when the noise distribution is rescaled Student's $t_{7}$ and satisfies assumptions A4 and A7. Under general conditions, which include A8, estimators (A)-(C) are asymptotically normal and $n^{1 / 2}$-consistent for $\left(\alpha_{00} / c, \alpha_{01} / c, \ldots, \alpha_{0 p} / c, \beta_{01}, \ldots, \beta_{0 q}\right)^{\prime}$, for different values of $c>0$. These techniques can, therefore, also be used to obtain $n^{1 / 2}$-consistent and asymptotically normal estimators of $\boldsymbol{\theta}_{0}$. For the noise distributions considered in Table 3.2, R-estimation with weight function $\lambda_{t_{7}}$ is more efficient than Laplace QML for all distributions except Laplace, and R-estimation uniformly dominates rescaled Student's $t_{7}$ QML and least absolute deviations with respect to asymptotic efficiency. AREs for R-estimation with weight function $\lambda_{t_{7}}$ with respect to Gaussian QML are also given in Table 3.2 (column (D)). Since, when compared to other techniques, R-estimation with weight function $\lambda_{t_{7}}$ performs well for light, medium, and heavier-tailed noise distributions, we recommend that it or R-estimation with a similar weight function be used in practice when the noise distribution is unknown. The weight function $\lambda_{t_{7}}$ is plotted in Figure 3.1, along with $\lambda_{N}$. Note that $\lambda_{t_{7}}(x)$ and $\lambda_{N}(x)$ are fairly similar except near $x=1$.

Remark 8: The Wilcoxon weight function $\lambda_{W}(x)=2 x-1$ is also shown in Figure 3.1. When estimating linear regression or linear time series model parameters via R-estimation, it is generally the recommended weight function when the noise distribution is unknown, since the corresponding R-estimators tend to be 


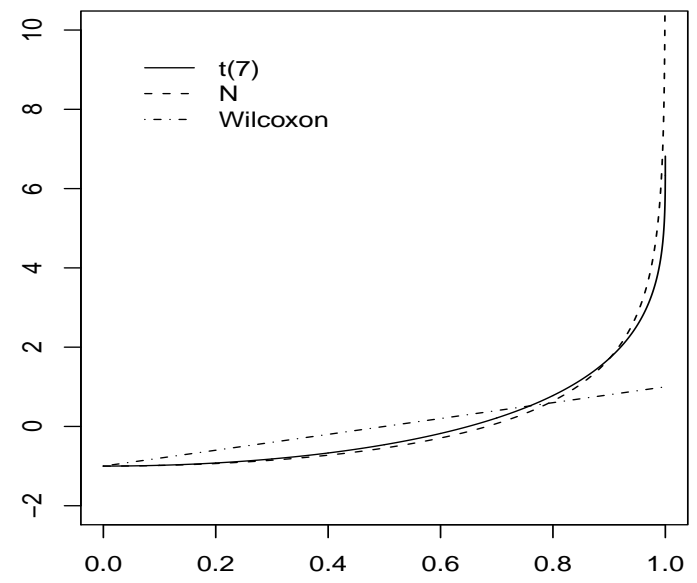

Figure 3.1: The weight functions $\lambda_{t_{7}}, \lambda_{N}$, and Wilcoxon weight function $\lambda_{W}$.

relatively efficient (see, for example, Hettmansperger and McKean, 1998, Andrews, Davis, and Breidt, 2007, and Andrews, 2008). In the case of linear model estimation, $\lambda_{W}$ is the optimal weight function when the noise distribution is logistic and, for R-estimation of GARCH model parameters, $\lambda_{W}$ is optimal when $\ln \left(Z_{t}^{2}\right)$ is logistic. R-estimation with the Wilcoxon function is not relatively efficient for GARCH models with commonly used noise distributions, however. To demonstrate, in Table 3.3, we give AREs for R-estimation with the Wilcoxon function with respect to R-estimation with weight function $\lambda_{t_{7}}$. All AREs are less than one.

Remark 9: For estimating $\boldsymbol{\eta}_{0}=\left(\alpha_{00}, \alpha_{01}, \ldots, \alpha_{0 p}, \beta_{01}, \ldots, \beta_{0 q}\right)^{\prime}$, an alternative to using Gaussian QML or ML with a noise distribution resembling the empirical distribution for R-estimation residuals is to estimate $\alpha_{00}$ via $\hat{\alpha}_{0}:=n^{-1} \sum_{t=p+1}^{n} \exp \left(\epsilon_{t}\left(\hat{\boldsymbol{\theta}}_{R}\right)\right)$ and then let $\hat{\boldsymbol{\eta}}=\left(\hat{\alpha}_{0}, \hat{\alpha}_{0} \hat{\theta}_{1, R}, \ldots, \hat{\alpha}_{0} \hat{\theta}_{p, R}, \hat{\theta}_{p+1, R}, \ldots, \hat{\theta}_{p+q, R}\right)^{\prime}$. Since

$$
\hat{\alpha}_{0}=n^{-1} \sum_{t=p+1}^{n} \exp \left(\epsilon_{t}\left(\hat{\boldsymbol{\theta}}_{R}\right)\right)=n^{-1} \sum_{t=p+1}^{n} \frac{X_{t}^{2}}{\tilde{\sigma}_{t}^{2}\left(\hat{\boldsymbol{\theta}}_{R}\right)}=n^{-1} \sum_{t=p+1}^{n} \alpha_{00} Z_{t}^{2} \frac{\sigma_{t}^{2} / \alpha_{00}}{\tilde{\sigma}_{t}^{2}\left(\hat{\boldsymbol{\theta}}_{R}\right)}
$$

and, using the proof of Lemma 5.8 in Berkes, Horváth, and Kokoszka (2003), it can be shown that the right-hand side of (3.4) equals $n^{-1} \sum_{t=p+1}^{n} \alpha_{00} Z_{t}^{2}+o_{p}(1), \hat{\alpha}_{0} \stackrel{P}{\rightarrow} \alpha_{00}$. By Theorem 3.1, $\hat{\boldsymbol{\theta}}_{R} \stackrel{P}{\rightarrow} \boldsymbol{\theta}_{0}=$ $\left(\alpha_{01} / \alpha_{00}, \ldots, \alpha_{0 p} / \alpha_{00}, \beta_{01}, \ldots, \beta_{0 q}\right)^{\prime}$, and so $\hat{\boldsymbol{\eta}}$ is consistent for $\boldsymbol{\eta}_{0}$. From Theorem 3.1 and Corollary 3.1, we have the limiting distributions for $\hat{\theta}_{i, R}, i=p+1, \ldots, p+q$, and when $\mathrm{E}\left\{Z_{t}^{4}\right\}<\infty$, it can be shown 


\begin{tabular}{|c|c|}
\hline Distribution for Noise $\left\{Z_{t}\right\}$ & ARE \\
\hline Laplace & 0.760 \\
logistic & 0.736 \\
$\mathrm{~N}(0,1)$ & 0.634 \\
$t_{3}$ & 0.911 \\
$t_{5}$ & 0.801 \\
$t_{7}$ & 0.753 \\
$t_{9}$ & 0.727 \\
$t_{12}$ & 0.703 \\
$t_{15}$ & 0.690 \\
$t_{20}$ & 0.676 \\
$t_{30}$ & 0.662 \\
\hline
\end{tabular}

Table 3.3: AREs for R-estimation with the Wilcoxon function with respect to $R$-estimation with weight function $\lambda_{t_{7}}$.

that $n^{1 / 2}\left(\hat{\alpha}_{0}-\alpha_{00}\right) \stackrel{d}{\rightarrow} \alpha_{00}\left(\boldsymbol{\xi}^{\prime} \mathrm{E}\left\{\partial \epsilon_{t}^{*}\left(\boldsymbol{\theta}_{0}\right) / \partial \boldsymbol{\theta}\right\}+\tilde{Y}\right)$ and, for $i \in\{1, \ldots, p\}, n^{1 / 2}\left(\hat{\alpha}_{0} \hat{\theta}_{i, R}-\alpha_{0 i}\right) \stackrel{d}{\rightarrow} \alpha_{00} \xi_{i}+$ $\alpha_{0 i}\left(\boldsymbol{\xi}^{\prime} \mathrm{E}\left\{\partial \epsilon_{t}^{*}\left(\boldsymbol{\theta}_{0}\right) / \partial \boldsymbol{\theta}\right\}+\tilde{Y}\right)$, with the random vector $\boldsymbol{\xi}$ as defined in equation $(3.2), \tilde{Y} \sim \mathrm{N}\left(0, \operatorname{Var}\left\{Z_{t}^{2}\right\}\right)$ independent of $\boldsymbol{\xi}$, and $\xi_{i}$ denotes the $i$ th element of $\boldsymbol{\xi}$. It follows that, if $\mathrm{A} 1-\mathrm{A} 8$ hold and $\mathrm{E}\left\{Z_{t}^{4}\right\}<\infty$, then $n^{1 / 2}\left(\hat{\alpha}_{0}-\alpha_{00}\right) \stackrel{d}{\rightarrow} \mathrm{N}\left(0, \alpha_{00}^{2}\left[\left(\mathrm{E}\left\{\partial \epsilon_{t}^{*}\left(\boldsymbol{\theta}_{0}\right) / \partial \boldsymbol{\theta}\right\}\right)^{\prime} \boldsymbol{\Sigma} \mathrm{E}\left\{\partial \epsilon_{t}^{*}\left(\boldsymbol{\theta}_{0}\right) / \partial \boldsymbol{\theta}\right\}+\operatorname{Var}\left\{Z_{t}^{2}\right\}\right]\right)$ and, for $i \in\{1, \ldots, p\}$, $n^{1 / 2}\left(\hat{\alpha}_{0} \hat{\theta}_{i, R}-\alpha_{0 i}\right) \stackrel{d}{\rightarrow} \mathrm{N}\left(0, \mathbf{C}_{i}^{\prime} \boldsymbol{\Sigma} \mathbf{C}_{i}+\alpha_{0 i}^{2} \operatorname{Var}\left\{Z_{t}^{2}\right\}\right)$, where

$$
\mathbf{C}_{i}:=(\underbrace{0, \ldots, 0}_{i-1 \text { times }} \alpha_{00}, \underbrace{0, \ldots, 0}_{p+q-i \text { times }})^{\prime}+\alpha_{0 i} \mathrm{E}\left\{\frac{\partial \epsilon_{t}^{*}\left(\boldsymbol{\theta}_{0}\right)}{\partial \boldsymbol{\theta}}\right\} .
$$

However, as is the case for Gaussian QMLEs of $\boldsymbol{\eta}_{0}$, when $\mathrm{E}\left\{Z_{t}^{4}\right\}=\infty$, the estimators $\hat{\alpha}_{0}$ and $\hat{\alpha}_{0} \hat{\theta}_{i, R}$, $i \in\{1, \ldots, p\}$, can be shown to have a rate of convergence slower than $n^{1 / 2}$, so this is not a robust technique.

\subsection{Order Selection and Interval Estimation}

To use Theorem 3.1 to make inferences about $\boldsymbol{\theta}_{0}$, estimates of $\tilde{K}$ and $\boldsymbol{\Gamma}$ are needed. Since $\lambda$ is determined by the practitioner, $\tilde{J}$ is known. For estimating $\boldsymbol{\Gamma}$, we propose using

$$
\hat{\boldsymbol{\Gamma}}_{n}:=\frac{1}{n} \sum_{t=p+1}^{n}\left[\frac{\partial \epsilon_{t}\left(\hat{\boldsymbol{\theta}}_{R}\right)}{\partial \boldsymbol{\theta}}-\left(\frac{1}{n} \sum_{s=p+1}^{n} \frac{\partial \epsilon_{s}\left(\hat{\boldsymbol{\theta}}_{R}\right)}{\partial \boldsymbol{\theta}}\right)\right]\left[\frac{\partial \epsilon_{t}\left(\hat{\boldsymbol{\theta}}_{R}\right)}{\partial \boldsymbol{\theta}}-\left(\frac{1}{n} \sum_{s=p+1}^{n} \frac{\partial \epsilon_{s}\left(\hat{\boldsymbol{\theta}}_{R}\right)}{\partial \boldsymbol{\theta}}\right)\right]^{\prime}
$$

with $\left\{\epsilon_{t}(\boldsymbol{\theta})\right\}$ as defined in (2.3). Since $\hat{\boldsymbol{\theta}}_{R} \stackrel{P}{\rightarrow} \boldsymbol{\theta}_{0}$, by the proofs of Lemmas 11 and 12 in Francq and Zakoïan (2007), $\hat{\boldsymbol{\Gamma}}_{n} \stackrel{P}{\rightarrow} \boldsymbol{\Gamma}$. A consistent estimator for $\tilde{K}$ is given in the following theorem.

Theorem 3.2. Consider the empirical distribution function $\hat{F}_{n}(x):=(n-p)^{-1} \sum_{t=p+1}^{n} I\left\{\epsilon_{t}\left(\hat{\boldsymbol{\theta}}_{R}\right) \leq x\right\}$ and 
the kernel density estimator

$$
\hat{f}_{n}(x):=\frac{1}{b_{n} n} \sum_{t=p+1}^{n} \kappa\left(\frac{\epsilon_{t}\left(\hat{\boldsymbol{\theta}}_{R}\right)-x}{b_{n}}\right)
$$

where $\kappa$ is a uniformly continuous, differentiable kernel density function on $\mathbb{R}$ such that $\kappa^{\prime}$ is uniformly continuous on $\mathbb{R}$ and $\int|x \ln | x||^{1 / 2}\left|\kappa^{\prime}(x)\right| d x<\infty$, and the bandwidth sequence $\left\{b_{n}\right\}$ is chosen so that $b_{n} \stackrel{P}{\rightarrow} 0$ and $b_{n}^{2} \sqrt{n} \stackrel{P}{\rightarrow} \infty$ as $n \rightarrow \infty$. If A1-A7 and either A8, A9 or A10 hold, then

$$
\begin{aligned}
\hat{K}_{n} & :=\int_{-\infty}^{\infty} \hat{f}_{n}(x) d \lambda\left(\hat{F}_{n}(x)\right)=\sum_{t=p+1}^{n} \hat{f}_{n}\left(\epsilon_{(t)}\left(\hat{\boldsymbol{\theta}}_{R}\right)\right)\left[\lambda\left(\frac{t-p}{n-p}\right)-\lambda\left(\frac{t-p-1}{n-p}\right)\right] \\
& \stackrel{P}{\rightarrow} \tilde{K} .
\end{aligned}
$$

Proof. Let $f_{\epsilon}$ and $F_{\epsilon}$ denote the density and distribution functions for $\epsilon_{t}=\ln \left(\alpha_{00} Z_{t}^{2}\right)$. By Lemma 5.5 in the Appendix, $\sup _{x \in \mathbb{R}}\left|\hat{f}_{n}(x)-f_{\epsilon}(x)\right| \stackrel{P}{\rightarrow} 0$ and, using the Glivenko-Cantelli theorem, $\sup _{x \in \mathbb{R}}\left|\hat{F}_{n}(x)-F_{\epsilon}(x)\right| \stackrel{P}{\rightarrow} 0$. As a result, $\hat{K}_{n} \stackrel{P}{\rightarrow} \int_{-\infty}^{\infty} f_{\epsilon}(x) d \lambda\left(F_{\epsilon}(x)\right)$ by the proof of Theorem 4.5.3 in Koul (2002). Since $f_{\epsilon}(x)=$ $f\left(x-\ln \left(\alpha_{00}\right)\right)$ and $F_{\epsilon}(x)=F\left(x-\ln \left(\alpha_{00}\right)\right), \tilde{K}=\int_{-\infty}^{\infty} f_{\epsilon}(x) d \lambda\left(F_{\epsilon}(x)\right)$, and so the proof is complete.

It follows that $\hat{\boldsymbol{\Sigma}}_{n}:=\tilde{J} \hat{K}_{n}^{-2} \hat{\boldsymbol{\Gamma}}_{n}^{-1}$ is consistent for $\boldsymbol{\Sigma}=\tilde{J} \tilde{K}^{-2} \boldsymbol{\Gamma}^{-1}$. Note that the Gaussian and Student's $t$ densities satisfy the conditions for the kernel density function $\kappa$ in Theorem 3.2.

For GARCH order selection, we can test null hypotheses of the form

$$
\mathrm{H}_{0}: \theta_{0 i_{1}}=\cdots=\theta_{0 i_{m}}=0
$$

with $1 \leq i_{1}<\cdots<i_{m} \leq p+q$. Following Corollary 3.1, a corresponding Wald test statistic is given by

$$
W_{n}:=n\left(\mathbf{W} \hat{\boldsymbol{\theta}}_{R}\right)^{\prime}\left(\mathbf{W} \hat{\boldsymbol{\Sigma}}_{n} \mathbf{W}^{\prime}\right)^{-1} \mathbf{W} \hat{\boldsymbol{\theta}}_{R}
$$

where $\mathbf{W}=\left[w_{j, k}\right]$ is the $m \times(p+q)$ matrix with $w_{\ell, i_{\ell}}=1$ for $\ell \in\{1, \ldots, m\}$ and $w_{j, k}=0$ otherwise. The limiting distribution for $W_{n}$ under $\mathrm{H}_{0}$ is given in the following theorem. This limiting distribution has a simple form when we are testing the nullity of just one parameter.

Theorem 3.3. Under (3.6), if $A 1-A 7$ and either $A 9$ or $A 10$ hold, then $W_{n} \stackrel{d}{\rightarrow} \tilde{W}:=\boldsymbol{\xi}^{\prime} \mathbf{W}^{\prime}\left(\mathbf{W} \boldsymbol{\Sigma} \mathbf{W}^{\prime}\right)^{-1} \mathbf{W} \boldsymbol{\xi}$ as $n \rightarrow \infty$, with $\boldsymbol{\xi}$ as defined in (3.2). If $m=1$ and $\theta_{0 j}>0$ for $j \neq i_{1}$, then $\tilde{W} \stackrel{d}{=} V^{2} I\{V \geq 0\}$ with $V \sim N(0,1)$. 
Proof. By Theorem 3.1, under these conditions, $n^{1 / 2} \mathbf{W} \hat{\boldsymbol{\theta}}_{R} \stackrel{d}{\rightarrow} \mathbf{W} \boldsymbol{\xi}$. Since $\hat{\boldsymbol{\Sigma}}_{n} \stackrel{P}{\rightarrow} \boldsymbol{\Sigma}$, it follows that $W_{n} \stackrel{d}{\rightarrow} \tilde{W}$. If $m=1$ and $\theta_{0 j}>0$ for $j \neq i_{1}$, following Remark $3, \mathbf{W} \boldsymbol{\xi}=\xi_{i_{1}}=Y_{i_{1}} I\left\{Y_{i_{1}} \geq 0\right\}$ with $Y_{i_{1}} \sim \mathrm{N}\left(0, \Sigma_{i_{1}, i_{1}}\right)$ $\left(\Sigma_{i_{1}, i_{1}}\right.$ represents element $i_{1}, i_{1}$ of $\left.\boldsymbol{\Sigma}\right)$, so $\tilde{W}=\Sigma_{i_{1}, i_{1}}^{-1} Y_{i_{1}}^{2} I\left\{Y_{i_{1}} \geq 0\right\} \stackrel{d}{=} V^{2} I\{V \geq 0\}$.

Therefore, assuming $\theta_{0 j}>0$ for $j \neq i_{1}$, we can reject the null hypothesis $\mathrm{H}_{0}: \theta_{0 i_{1}}=0$ at level of significance $\alpha<1 / 2$ if $W_{n}>\chi_{1,1-2 \alpha}^{2}$, where $\chi_{1,1-2 \alpha}^{2}$ represents the $1-2 \alpha$ quantile of the chi-squared distribution with one degree of freedom. Finding a critical value for testing the nullity of multiple parameters $\theta_{0 i_{1}}, \ldots, \theta_{0 i_{m}}$ is not as simple, since, in general, the distribution for the limiting random variable $\tilde{W}$ under $\mathrm{H}_{0}$ depends on the values of the other model parameters and the distribution for the iid noise $\left\{Z_{t}\right\}$. However, one can examine the distribution for $W_{n}$ under $\mathrm{H}_{0}$ in practice by simulating GARCH series of length $n$ with parameter vector $\boldsymbol{\theta}^{*}=\left(\theta_{1}^{*}, \ldots, \theta_{p+q}^{*}\right)^{\prime}$, with $\theta_{j}^{*}=0$ for $j \in\left\{i_{1}, \ldots, i_{m}\right\}$ and $\theta_{j}^{*}=\hat{\theta}_{j, R}$ otherwise, and iid noise following the empirical distribution for R-estimation residuals, and then obtaining the corresponding values for $W_{n}$.

Test statistics for testing the nullity of GARCH model parameters using Gaussian QMLEs are considered in Francq and Zakoïan (2009). Limiting results in Francq and Zakoïan (2009) require $\mathrm{E}\left\{Z_{t}^{4}\right\}<\infty$, however. In contrast, when A9 holds, higher-order moment conditions are not required for the limiting result in Theorem 3.3.

Although parameter estimation for the $\operatorname{ARCH}(p)$ model with $\theta_{01}=\cdots=\theta_{0 p}=0$ is not considered in this paper since A2 is not satisfied, our results can be used to show that, under $\mathrm{H}_{0}: \theta_{01}=\cdots=\theta_{0 p}=0$, if $E\left\{Z_{t}^{4}\right\}<\infty$, then $W_{n} \stackrel{d}{\rightarrow} \tilde{W}=\boldsymbol{\xi}^{\prime} \boldsymbol{\Sigma}^{-1} \boldsymbol{\xi}$, where, in this case, $\boldsymbol{\xi}=\left(Y_{1} I\left\{Y_{1} \geq 0\right\}, \ldots, Y_{p} I\left\{Y_{p} \geq 0\right\}\right)^{\prime}$, $\mathbf{Y}=\left(Y_{1}, \ldots, Y_{p}\right)^{\prime} \sim \mathrm{N}(\mathbf{0}, \boldsymbol{\Sigma})$, and $\boldsymbol{\Sigma}=\tilde{J} \tilde{K}^{-2}\left(\operatorname{Var}\left\{Z_{t}^{2}\right\}\right)^{-1} \mathbf{I}(\mathbf{I}$ represents the identity matrix $)$. It follows that, under these conditions, $W_{n} \stackrel{d}{\rightarrow} V_{1}^{2} I\left\{V_{1} \geq 0\right\}+\cdots+V_{p}^{2} I\left\{V_{p} \geq 0\right\}$, where $V_{1}, \ldots, V_{p}$ are iid $\mathrm{N}(0,1)$. Consequently, R-estimation and the Wald test statistic (3.7) can be used to identify ARCH-type conditional heteroskedasticity in an observed time series. However, because $\mathrm{E}\left\{Z_{t}^{4}\right\}<\infty$ is required, this technique is just as robust as more traditional Gaussian likelihood-based techniques (see Francq and Zakö̈an, 2009). Therefore, in practice, the rank-based Wald test statistic is most useful for choosing between GARCH models of different orders that are under consideration for an observed conditionally heteroskedastic series. Once appropriate GARCH model orders $p$ and $q$ have been identified, confidence intervals for the elements of $\boldsymbol{\theta}_{0}$ 
can be obtained using the limiting normal result in Corollary 3.1 and the consistent estimate $\hat{\boldsymbol{\Sigma}}_{n}$ of $\boldsymbol{\Sigma}$.

\section{Numerical Results}

\subsection{Simulation Study}

In this section, we give the results of a simulation study to assess the quality of the asymptotic approximations for finite samples. First, for each of 1000 replicates, we simulated a $\operatorname{GARCH}(1,1)$ series with parameters $\alpha_{00}=0.01, \alpha_{01}=0.5$, and $\beta_{01}=0.4$, and found the R-estimate $\hat{\boldsymbol{\theta}}_{R}$ of $\boldsymbol{\theta}_{0}=(0.5 / 0.01,0.4)^{\prime}=(50,0.4)^{\prime}$ by minimizing $D_{n}$ in (2.5). To reduce the possibility of the optimizer getting trapped at local minima, we used 100 starting values for each replicate. Starting values for $\alpha_{1}$ and $\beta_{1}=\theta_{2}$, with $\alpha_{1}+\beta_{1}<1$, were randomly chosen, and then, since $\alpha_{1}+\beta_{1}<1$ implies $\operatorname{Var}\left\{X_{t}\right\}=\alpha_{0} /\left(1-\alpha_{1}-\beta_{1}\right)$, we used the values of $\alpha_{1} /\left[s_{X}^{2}\left(1-\alpha_{1}-\beta_{1}\right)\right]$, where $s_{X}^{2}$ represents sample variance for $\left\{X_{t}\right\}_{t=1}^{n}$, for the starting values of $\theta_{1}=\alpha_{1} / \alpha_{0}$. We evaluated $D_{n}$ at each of the 100 candidate values and then reduced the collection of initial values to the three with the smallest values of $D_{n}$. Using these three initial values as starting points, we found optimized values by implementing the Nelder-Mead algorithm (Nelder and Mead, 1965). The optimized value for which $D_{n}$ was smallest was chosen to be $\hat{\boldsymbol{\theta}}_{R}$. By Corollary 3.1, in this $\operatorname{GARCH}(1,1)$ case, $\hat{\boldsymbol{\theta}}_{R}$ is asymptotically normal with mean $\boldsymbol{\theta}_{0}$ and covariance matrix $n^{-1} \boldsymbol{\Sigma}=n^{-1} \tilde{J} \tilde{K}^{-2} \boldsymbol{\Gamma}^{-1}$, with

$$
\boldsymbol{\Gamma}=\operatorname{Var}\left\{\frac{\partial \epsilon_{t}^{*}\left(\boldsymbol{\theta}_{0}\right)}{\partial \boldsymbol{\theta}}\right\}=\operatorname{Var}\left\{\left[\begin{array}{c}
\alpha_{00}\left(\sum_{j=0}^{\infty} \beta_{01}^{j} X_{t-1-j}^{2}\right) / \sigma_{t}^{2} \\
\left(\sum_{j=0}^{\infty} \beta_{01}^{j} \sigma_{t-1-j}^{2}\right) / \sigma_{t}^{2}
\end{array}\right]\right\} .
$$

Confidence intervals for the elements of $\boldsymbol{\theta}_{0}$ were, therefore, constructed using the asymptotic normality and $\hat{\boldsymbol{\Sigma}}_{n}$, the consistent estimator of $\boldsymbol{\Sigma}$. For the kernel density estimator (3.5), we used the standard Gaussian kernel density function and, because of its recommendation in Silverman (1986, page 48), we used bandwidth $b_{n}=0.9 n^{-1 / 5} \min \left\{s_{\epsilon}, I Q R_{\epsilon} / 1.34\right\}$, where $s_{\epsilon}$ and $I Q R_{\epsilon}$ represent sample standard deviation and interquartile range for $\left\{\epsilon_{t}\left(\hat{\boldsymbol{\theta}}_{R}\right)\right\}_{t=p+1}^{n}$.

Results of these simulations, for $\mathrm{N}(0,1)$ and rescaled $t_{3}$ noise, and weight functions $\lambda_{t_{7}}(x)=\left[7\left\{F_{t_{7}}^{-1}((x+\right.\right.$ $\left.1) / 2)\}^{2}-5\right] /\left[\left\{F_{t_{7}}^{-1}((x+1) / 2)\right\}^{2}+5\right]$ and $\lambda_{W}(x)=2 x-1$, are given in Tables 4.1 and 4.2. We show the empirical means, standard deviations, and percent coverages of nominal $95 \%$ confidence intervals for the 


\begin{tabular}{|c|c|c|c|c|c|}
\hline$n$ & Asymp & $\begin{array}{l}\text { tic } \\
\text { std. dev. } \\
\left(\mathrm{N}(0,1), t_{3}\right)\end{array}$ & $\begin{array}{c}\text { mean } \\
\left(\mathrm{N}(0,1), t_{3}\right)\end{array}$ & $\begin{array}{c}\text { Empirical } \\
\text { std. dev. } \\
\left(\mathrm{N}(0,1), t_{3}\right)\end{array}$ & $\begin{array}{l}\text { \% coverage } \\
\left(\mathrm{N}(0,1), t_{3}\right)\end{array}$ \\
\hline 250 & $\theta_{1}=\alpha_{1} / \alpha_{0}=50$ & $19.6,25.3$ & $48.3,52.0$ & $20.2,28.6$ & $85.4,89.0$ \\
\hline & $\theta_{2}=\beta_{1}=0.4$ & $0.104,0.158$ & $0.378, \quad 0.369$ & $0.124, \quad 0.178$ & $92.6,91.0$ \\
\hline 500 & $\theta_{1}=\alpha_{1} / \alpha_{0}=50$ & $13.9,17.9$ & $50.3,49.8$ & $14.4,17.8$ & $91.7,91.0$ \\
\hline & $\theta_{2}=\beta_{1}=0.4$ & $0.073, \quad 0.112$ & $0.389,0.385$ & $0.079, \quad 0.122$ & $94.8,95.1$ \\
\hline 2000 & $\theta_{1}=\alpha_{1} / \alpha_{0}=50$ & $6.93,8.94$ & $49.8,50.4$ & $7.00,8.83$ & $94.1,95.6$ \\
\hline & $\theta_{2}=\beta_{1}=0.4$ & $0.037,0.056$ & $0.398,0.399$ & $0.038,0.057$ & $96.6,97.6$ \\
\hline
\end{tabular}

Table 4.1: Empirical means, standard deviations, and percent coverages of nominal $95 \%$ confidence intervals for R-estimates of GARCH model parameters. The $N(0,1)$ and rescaled $t_{3}$ noise distributions and weight function $\lambda_{t_{7}}(x)=\left[7\left\{F_{t_{7}}^{-1}((x+1) / 2)\right\}^{2}-5\right] /\left[\left\{F_{t_{7}}^{-1}((x+1) / 2)\right\}^{2}+5\right]$ were used.

\begin{tabular}{|c|c|c|c|c|c|}
\hline$n$ & Asymp & $\begin{array}{l}\text { stic } \\
\left(\mathrm{N}(0,1), t_{3}\right)\end{array}$ & $\begin{array}{c}\text { mean } \\
\left(\mathrm{N}(0,1), t_{3}\right)\end{array}$ & $\begin{array}{c}\text { Empirical } \\
\text { std. dev. } \\
\left(\mathrm{N}(0,1), t_{3}\right)\end{array}$ & $\begin{array}{l}\text { \% coverage } \\
\left(\mathrm{N}(0,1), t_{3}\right)\end{array}$ \\
\hline \multirow[t]{2}{*}{250} & $\theta_{1}=\alpha_{1} / \alpha_{0}=50$ & $24.6,26.5$ & $52.0,52.9$ & $32.9,30.6$ & $87.5,88.5$ \\
\hline & $\theta_{2}=\beta_{1}=0.4$ & $0.130,0.166$ & $0.375,0.377$ & $0.148,0.185$ & $93.2,92.1$ \\
\hline \multirow[t]{2}{*}{500} & $\theta_{1}=\alpha_{1} / \alpha_{0}=50$ & $17.4,18.7$ & $51.2,51.9$ & $19.5,21.3$ & $91.1,92.9$ \\
\hline & $\theta_{2}=\beta_{1}=0.4$ & $0.092,0.117$ & $0.389, \quad 0.395$ & $0.102,0.120$ & $94.2,96.9$ \\
\hline \multirow[t]{2}{*}{2000} & $\theta_{1}=\alpha_{1} / \alpha_{0}=50$ & $8.70,9.37$ & $50.0,50.7$ & $8.79,9.57$ & $94.4,95.5$ \\
\hline & $\theta_{2}=\beta_{1}=0.4$ & $0.046,0.059$ & $0.397,0.395$ & $0.046,0.060$ & $97.6,96.7$ \\
\hline
\end{tabular}

Table 4.2: Empirical means, standard deviations, and percent coverages of nominal $95 \%$ confidence intervals for R-estimates of GARCH model parameters. The $N(0,1)$ and rescaled $t_{3}$ noise distributions and Wilcoxon weight function $\lambda_{W}(x)=2 x-1$ were used.

R-estimates of $\boldsymbol{\theta}_{0}$. The asymptotic means and standard deviations are also given in Tables 4.1 and 4.2 . We see that the R-estimates appear nearly unbiased, and the asymptotic standard deviations fairly accurately reflect the true variability of the estimates, so R-estimation with weight function $\lambda_{t_{7}}$ is more precise than R-estimation with the Wilcoxon weight function. We also see that the confidence interval coverages are close to the nominal $95 \%$ level, especially when $n=2000$. Normal probability plots show that the R-estimates are approximately normal, particularly for large $n$.

We also ran simulations to assess the accuracy of the asymptotic approximations in the case of one null parameter value, by fitting $\operatorname{GARCH}(2,1)$ and $\operatorname{GARCH}(1,2)$ models to $\operatorname{GARCH}(1,1)$ series with $\boldsymbol{\theta}_{0}=(50,0.4)^{\prime}$. When $\theta_{0 j}=0$, we obtained the Wald statistic (3.7) for testing $\mathrm{H}_{0}: \theta_{0 j}=0$ and compared it to $\chi_{1,0.9}^{2}=2.706$, the level 0.05 critical value for testing the nullity of one parameter assuming all other parameters are positive. 


\begin{tabular}{|c|l|ccc|}
\hline & & \multicolumn{4}{|c|}{ Empirical } \\
$n$ & & mean & std. dev. & \% Wald stats. $>2.706$ \\
\hline 250 & $\theta_{1}=\alpha_{1} / \alpha_{0}=50$ & $41.7,42.3$ & $21.2,26.3$ & \\
& $\theta_{2}=\alpha_{2} / \alpha_{0}=0$ & $7.15,10.6$ & $8.97,14.5$ & $16.2,7.0$ \\
& $\theta_{3}=\beta_{1}=0.4$ & $0.296,0.273$ & $0.171,0.207$ & \\
\hline 500 & $\theta_{1}=\alpha_{1} / \alpha_{0}=50$ & $43.9,43.8$ & $15.5,19.0$ & \\
& $\theta_{2}=\alpha_{2} / \alpha_{0}=0$ & $4.87,7.62$ & $6.16,9.58$ & $13.7,11.4$ \\
& $\theta_{3}=\beta_{1}=0.4$ & $0.326,0.307$ & $0.124,0.164$ & \\
\hline 2000 & $\theta_{1}=\alpha_{1} / \alpha_{0}=50$ & $46.9,46.6$ & $7.72,10.0$ & \\
& $\theta_{2}=\alpha_{2} / \alpha_{0}=0$ & $2.66,3.79$ & $3.35,5.00$ & \\
& $\theta_{3}=\beta_{1}=0.4$ & $0.369,0.359$ & $0.053,0.082$ & \\
\hline 250 & $\theta_{1}=\alpha_{1} / \alpha_{0}=50$ & $51.2,52.0$ & $22.5,28.9$ & \\
& $\theta_{2}=\beta_{1}=0.4$ & $0.297,0.276$ & $0.148,0.188$ & \\
& $\theta_{3}=\beta_{2}=0$ & $0.060,0.080$ & $0.095,0.127$ & $9.9,7.1$ \\
\hline 500 & $\theta_{1}=\alpha_{1} / \alpha_{0}=50$ & $49.9,51.6$ & $14.5,18.8$ & \\
& $\theta_{2}=\beta_{1}=0.4$ & $0.337,0.312$ & $0.109,0.144$ & \\
& $\theta_{3}=\beta_{2}=0$ & $0.039,0.059$ & $0.065,0.094$ & $7.9,8.3$ \\
\hline 2000 & $\theta_{1}=\alpha_{1} / \alpha_{0}=50$ & $50.1,50.4$ & $7.11,9.13$ & \\
& $\theta_{2}=\beta_{1}=0.4$ & $0.368,0.356$ & $0.054,0.081$ & \\
& $\theta_{3}=\beta_{2}=0$ & $0.020,0.030$ & $0.030,0.044$ & $5.9,6.5$ \\
\hline
\end{tabular}

Table 4.3: Empirical means, standard deviations, and the percentages of Wald statistics above 2.706 for $R$ estimates of GARCH model parameters. The $N(0,1)$ and rescaled $t_{3}$ noise distributions and weight function $\lambda_{t_{7}}(x)=\left[7\left\{F_{t_{7}}^{-1}((x+1) / 2)\right\}^{2}-5\right] /\left[\left\{F_{t_{7}}^{-1}((x+1) / 2)\right\}^{2}+5\right]$ were used.

Empirical means and standard deviations for the estimates, and the percentages of Wald statistics above 2.706 are given in Tables 4.3 and 4.4. The R-estimators appear consistent in both the GARCH(2,1) and GARCH(1,2) cases, and the test sizes are close to the nominal 0.05 level, particularly when $n$ is large.

\subsection{GARCH Modeling}

In Figure 4.1(a), we give the daily log-returns for the Japanese yen to U.S. dollar exchange rate for January 4, 1993-December 31, 2002. Sample autocorrelation functions for these data, $\left\{X_{t}\right\}_{t=1}^{2514}$, and their absolute values and squares are given in Figure 4.1(b)-(d) with the bounds $\pm 1.96 / \sqrt{2514}$, approximate $95 \%$ confidence bounds for the sample correlations assuming the observations are iid (see, for example, Brockwell and Davis, 1991, Section 7.2). Since the log-returns $\left\{X_{t}\right\}$ exhibit volatility clustering and appear uncorrelated but dependent, a GARCH model appears appropriate for this series. To find a suitable fitted model, we replaced the seventeen values of $X_{t}=0$ with $X_{t}=10^{-8}$ (a very slight alteration of the series which allows us to assume 


\begin{tabular}{|c|l|ccc|}
\hline & & \multicolumn{4}{|c|}{ Empirical } \\
$n$ & & mean & std. dev. & \% Wald stats. $>2.706$ \\
& & $\left(\mathrm{~N}(0,1), t_{3}\right)$ & $\left(\mathrm{N}(0,1), t_{3}\right)$ & $\left(\mathrm{N}(0,1), t_{3}\right)$ \\
\hline 250 & $\theta_{1}=\alpha_{1} / \alpha_{0}=50$ & $41.6,43.7$ & $26.2,29.9$ & \\
& $\theta_{2}=\alpha_{2} / \alpha_{0}=0$ & $8.48,10.8$ & $10.9,14.0$ & $7.9,3.4$ \\
& $\theta_{3}=\beta_{1}=0.4$ & $0.279,0.286$ & $0.191,0.219$ & \\
\hline 500 & $\theta_{1}=\alpha_{1} / \alpha_{0}=50$ & $43.6,45.2$ & $18.7,20.9$ & \\
& $\theta_{2}=\alpha_{2} / \alpha_{0}=0$ & $6.44,7.82$ & $8.17,10.2$ & $13.2,9.1$ \\
& $\theta_{3}=\beta_{1}=0.4$ & $0.314,0.308$ & $0.146,0.169$ & \\
\hline 2000 & $\theta_{1}=\alpha_{1} / \alpha_{0}=50$ & $46.8,46.5$ & $9.61,10.2$ & \\
& $\theta_{2}=\alpha_{2} / \alpha_{0}=0$ & $2.91,3.95$ & $4.00,5.48$ & \\
& $\theta_{3}=\beta_{1}=0.4$ & $0.365,0.360$ & $0.071,0.083$ & \\
\hline 250 & $\theta_{1}=\alpha_{1} / \alpha_{0}=50$ & $54.6,56.0$ & $42.7,33.7$ & \\
& $\theta_{2}=\beta_{1}=0.4$ & $0.288,0.271$ & $0.167,0.201$ & \\
& $\theta_{3}=\beta_{2}=0$ & $0.078,0.090$ & $0.124,0.141$ & \\
\hline 500 & $\theta_{1}=\alpha_{1} / \alpha_{0}=50$ & $51.3,52.3$ & $18.5,19.9$ & \\
& $\theta_{2}=\beta_{1}=0.4$ & $0.326,0.318$ & $0.128,0.156$ & \\
& $\theta_{3}=\beta_{2}=0$ & $0.050,0.063$ & $0.083,0.100$ & $8.0,8.6$ \\
\hline 2000 & $\theta_{1}=\alpha_{1} / \alpha_{0}=50$ & $50.5,50.6$ & $8.84,9.44$ & \\
& $\theta_{2}=\beta_{1}=0.4$ & $0.363,0.351$ & $0.070,0.085$ & \\
& $\theta_{3}=\beta_{2}=0$ & $0.026,0.032$ & $0.039,0.047$ & $6.0,7.1$ \\
\hline
\end{tabular}

Table 4.4: Empirical means, standard deviations, and the percentages of Wald statistics above 2.706 for $R$ estimates of GARCH model parameters. The $N(0,1)$ and rescaled $t_{3}$ noise distributions and Wilcoxon weight function $\lambda_{W}(x)=2 x-1$ were used. 
(a) Log-Returns

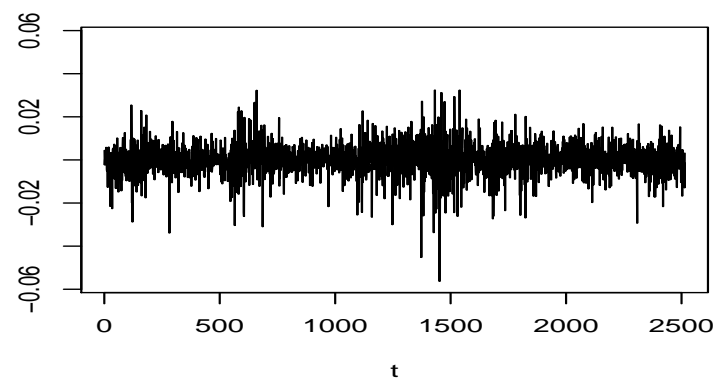

(c) ACF of Absolute Values

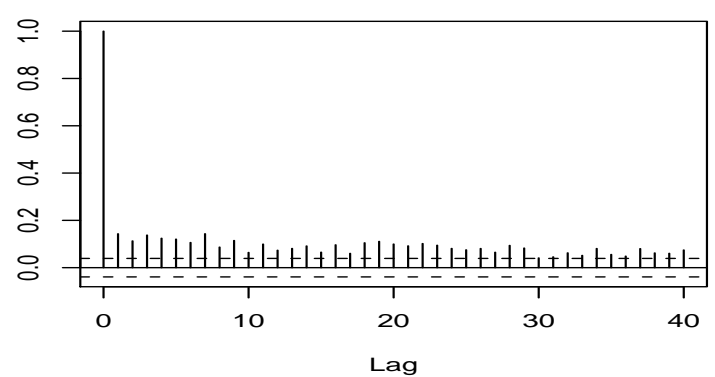

(b) ACF

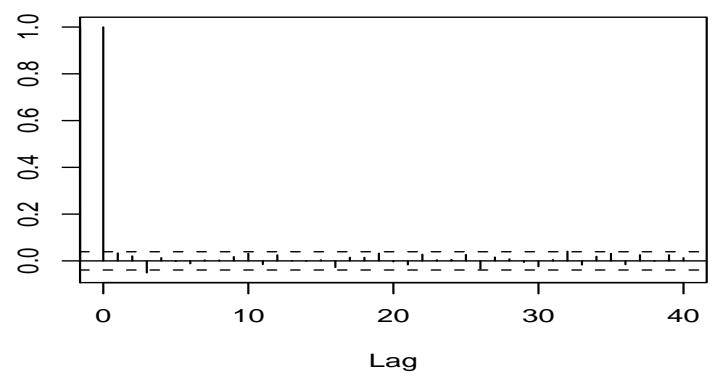

(d) ACF of Squares

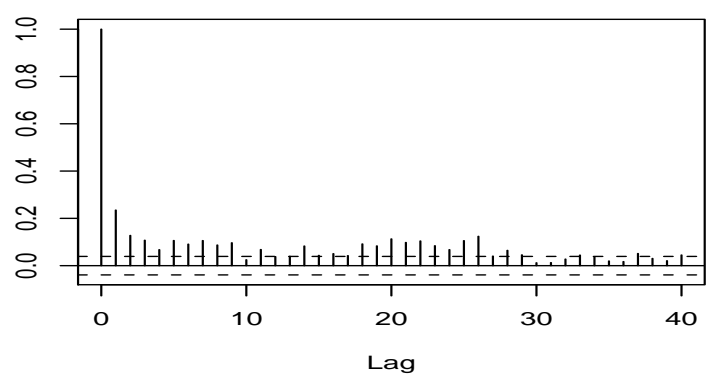

Figure 4.1: (a) The daily log-returns for the Japanese yen to U.S. dollar exchange rate for January 4, 1993December 31, 2002, with sample autocorrelation functions for (b) the log-returns, (c) their absolute values, and (d) their squares. 
(a) ACF of Absolute Values

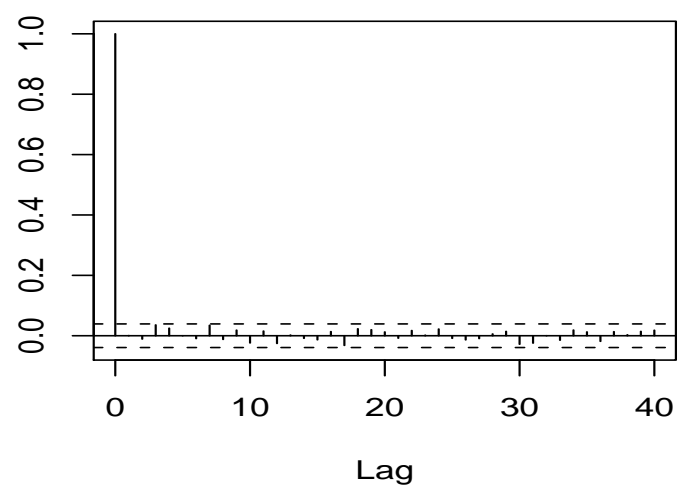

(b) ACF of Squares

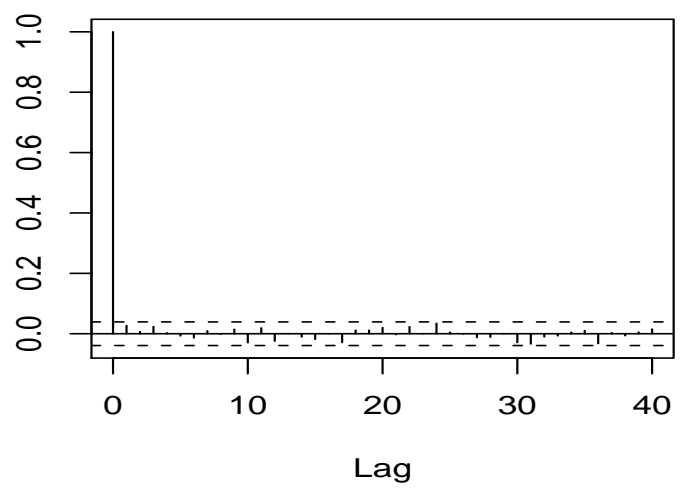

Figure 4.2: Sample autocorrelation functions for the (a) absolute values and (b) squares of the GARCH(1,1) residuals obtained via $R$-estimation.

A3 and compute $\left\{\ln \left(X_{t}^{2}\right)\right\}$ ), and fit low order ARCH/GARCH models to the data via R-estimation with weight function $\lambda_{t_{7}}(x)=\left[7\left\{F_{t_{7}}^{-1}((x+1) / 2)\right\}^{2}-5\right] /\left[\left\{F_{t_{7}}^{-1}((x+1) / 2)\right\}^{2}+5\right]$. ARCH(1) and $\operatorname{ARCH}(2)$ models were not appropriate, since those residuals were dependent, but, as is often the case with log-returns series, $\operatorname{GARCH}(1,1)$ residuals appeared independent. To demonstrate, in Figure 4.2, we give sample autocorrelation functions for the absolute values and squares of the residuals $\left\{X_{t} / \sqrt{\tilde{\sigma}_{t}^{2}\left(\hat{\boldsymbol{\theta}}_{R}\right)}\right\}$ from the $\operatorname{GARCH}(1,1)$ fitted model; note that the values of $\left\{X_{t} / \sqrt{\tilde{\sigma}_{t}^{2}\left(\hat{\boldsymbol{\theta}}_{R}\right)}\right\}$ resemble $\left\{X_{t} / \sqrt{\sigma_{t}^{2} / \alpha_{00}}\right\}=\left\{\sqrt{\alpha_{00}} Z_{t}\right\}$ when $\hat{\boldsymbol{\theta}}_{R}$ is close to the true parameter vector $\boldsymbol{\theta}_{0}$. The corresponding R-estimates for the $\operatorname{GARCH}(1,1)$ parameter values are $\hat{\theta}_{1}=\widehat{\alpha_{1} / \alpha_{0}}=45274$ and $\hat{\theta}_{2}=\hat{\beta}_{1}=0.9388$, with approximate $95 \%$ confidence intervals $(20793,69755)$ and $(0.9170,0.9606)$. Higher order $\operatorname{GARCH}(2,1)$ and $\operatorname{GARCH}(1,2)$ models were also considered, but low values of the Wald test statistic led us to fail to reject the null hypotheses $\mathrm{H}_{0}: \alpha_{02} / \alpha_{00}=0$ and $\mathrm{H}_{0}: \beta_{02}=0$ at the 0.05 level of significance. This analysis, therefore, indicates that a GARCH(1,1) model is suitable for the exchange rate log-returns.

We then considered using ML to fit a $\operatorname{GARCH}(1,1)$ model to the data and obtain individual estimates for $\alpha_{00}$ and $\alpha_{01}$. A kernel estimate of the density for the standardized $\operatorname{GARCH}(1,1)$ residuals, ie. $\left\{X_{t} / \sqrt{\tilde{\sigma}_{t}^{2}\left(\hat{\boldsymbol{\theta}}_{R}\right)}\right\}$ standardized to have variance one, is given in Figure 4.3(a), along with the $\mathrm{N}(0,1)$ density. Since the distribution for the residuals appears roughly symmetric, but more peaked and heavier- 
(a)

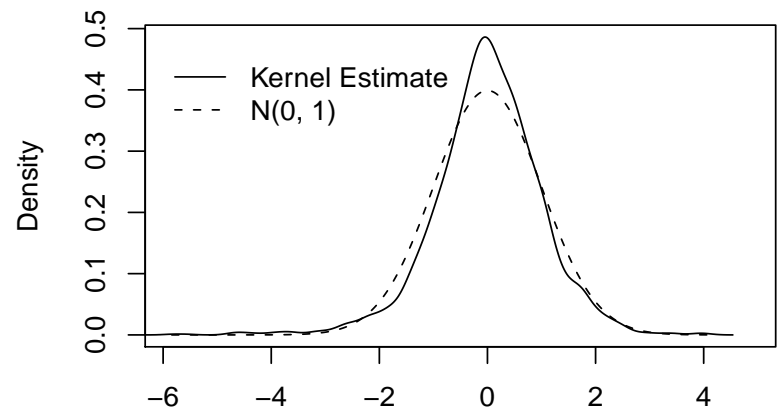

(b)

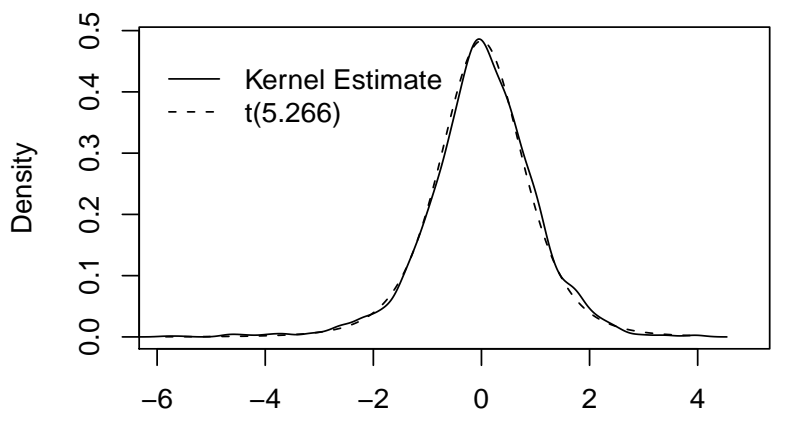

Figure 4.3: Kernel estimate of the density for the standardized $\operatorname{GARCH}(1,1)$ residuals with $(a)$ the $N(0,1)$ density function and (b) the rescaled $t_{5.266}$ density function.

tailed than Gaussian, we considered modeling the $\operatorname{GARCH}(1,1)$ noise distribution as rescaled Student's $t$. The ML estimate of degrees of freedom is 5.266 and, in Figure 4.3(b), it can be seen that the kernel density estimate for the standardized residuals and the $t_{5.266}$ density are close, so it appears reasonable to model the log-returns series as $\operatorname{GARCH}(1,1)$ with iid rescaled $t_{5.266}$ noise. Corresponding ML estimates of the model parameters are $\hat{\alpha}_{0}=9.632 \times 10^{-7}, \hat{\alpha}_{1}=0.04356$, and $\hat{\beta}_{1}=0.9393$, and, using the theory of Berkes and Horváth (2004), approximate 95\% confidence intervals for the parameter values are $\left(4.310 \times 10^{-7}, 14.953 \times 10^{-7}\right),(0.02663,0.06050)$, and $(0.9176,0.9610)$. Note that the ML estimate for $\beta_{01}$ and the corresponding confidence interval are nearly the same as those obtained via R-estimation, even though no specific distributional information was used for R-estimation. Also, $\hat{\alpha}_{1} / \hat{\alpha}_{0}=45231$, which is quite close to the R-estimate of $\theta_{01}=\alpha_{01} / \alpha_{00}$.

Finally, to verify that the rescaled $t_{5.266}$ distribution is suitable for the fitted $\operatorname{GARCH}(1,1)$ noise process, we used the ML residuals and the Kolmogorov-Smirnov test described in Koul and Ling (2006) to test this null hypothesis. The test statistic $K_{n}$ equals 1.194 and, via simulation, we found a corresponding p-value of 0.409. We, therefore, failed to reject $\mathrm{H}_{0}$ (the noise distribution is $t_{5.266}$ ) at the 0.05 level of significance, and so this test result indicates that the rescaled $t_{5.266}$ distribution is appropriate. 


\section{Appendix}

This section contains proofs of the lemmas used to establish Theorems 3.1 and 3.2. Assume conditions A1-A7 and either A8, A9 or A10 hold throughout. To begin, note that following (2.3)-(2.4), the first partial derivatives for the residuals $\left\{\epsilon_{t}(\boldsymbol{\theta})\right\}_{t=1}^{n}$ are given by $\partial \epsilon_{t}(\boldsymbol{\theta}) / \partial \theta_{i}=-\left[\partial \tilde{\sigma}_{t}^{2}(\boldsymbol{\theta}) / \partial \theta_{i}\right] / \tilde{\sigma}_{t}^{2}(\boldsymbol{\theta}), i \in\{1, \ldots, p+q\}$, with

$$
\frac{\partial \tilde{\sigma}_{t}^{2}(\boldsymbol{\theta})}{\partial \theta_{i}}= \begin{cases}0, & t \leq p, \\ X_{t-i}^{2}+\sum_{j=1}^{q} \theta_{p+j}\left(\partial \tilde{\sigma}_{t-j}^{2}(\boldsymbol{\theta}) / \partial \theta_{i}\right), & t \in\{p+1, \ldots, n\}, i \in\{1, \ldots, p\}, \\ \tilde{\sigma}_{t+p-i}^{2}(\boldsymbol{\theta})+\sum_{j=1}^{q} \theta_{p+j}\left(\partial \tilde{\sigma}_{t-j}^{2}(\boldsymbol{\theta}) / \partial \theta_{i}\right), & t \in\{p+1, \ldots, n\}, i \in\{p+1, \ldots, p+q\} .\end{cases}
$$

And we let

$$
\frac{\overline{\partial \epsilon(\boldsymbol{\theta})}}{\partial \boldsymbol{\theta}}=\frac{1}{n-p} \sum_{t=p+1}^{n} \frac{\partial \epsilon_{t}(\boldsymbol{\theta})}{\partial \boldsymbol{\theta}} \quad \text { and } \quad \overline{\frac{\partial \epsilon^{*}(\boldsymbol{\theta})}{\partial \boldsymbol{\theta}}}=\frac{1}{n-p} \sum_{t=p+1}^{n} \frac{\partial \epsilon_{t}^{*}(\boldsymbol{\theta})}{\partial \boldsymbol{\theta}}
$$

(recall that the partial derivatives $\partial \epsilon_{t}^{*}(\boldsymbol{\theta}) / \partial \theta_{i}, i \in\{1, \ldots, p+q\}$, are given in (3.1)).

Lemma 5.1. As $n \rightarrow \infty$,

$$
\frac{1}{\sqrt{n}} \sum_{t=p+1}^{n} \lambda\left(F_{\epsilon}\left(\epsilon_{t}\right)\right)\left[\frac{\partial \epsilon_{t}\left(\boldsymbol{\theta}_{0}\right)}{\partial \boldsymbol{\theta}}-\overline{\frac{\partial \epsilon\left(\boldsymbol{\theta}_{0}\right)}{\partial \boldsymbol{\theta}}}\right] \stackrel{d}{\rightarrow} \mathbf{N} \sim N(\mathbf{0}, \tilde{J} \boldsymbol{\Gamma}),
$$

where $\epsilon_{t}=\ln \left(\alpha_{00} Z_{t}^{2}\right)$ and $F_{\epsilon}$ represents the distribution function for $\epsilon_{t}$.

Proof. By the proof of Lemma 12 in Francq and Zakoïan (2007), $n^{-1 / 2} \sum_{t=p+1}^{n}\left|\partial \epsilon_{t}\left(\boldsymbol{\theta}_{0}\right) / \partial \theta_{i}-\partial \epsilon_{t}^{*}\left(\boldsymbol{\theta}_{0}\right) / \partial \theta_{i}\right| \stackrel{P}{\rightarrow}$ 0 for any $i \in\{1, \ldots, p+q\}$. It, therefore, suffices to show that

$$
\begin{aligned}
& \frac{1}{\sqrt{n}} \sum_{t=p+1}^{n} \lambda\left(F_{\epsilon}\left(\epsilon_{t}\right)\right)\left[\frac{\partial \epsilon_{t}^{*}\left(\boldsymbol{\theta}_{0}\right)}{\partial \boldsymbol{\theta}}-\frac{\overline{\partial \epsilon^{*}\left(\boldsymbol{\theta}_{0}\right)}}{\partial \boldsymbol{\theta}}\right] \\
&=\frac{1}{\sqrt{n}} \sum_{t=p+1}^{n}\left[\lambda\left(F_{\epsilon}\left(\epsilon_{t}\right)\right)-\mathrm{E}\left\{\lambda\left(F_{\epsilon}\left(\epsilon_{t}\right)\right)\right\}\right]\left[\frac{\partial \epsilon_{t}^{*}\left(\boldsymbol{\theta}_{0}\right)}{\partial \boldsymbol{\theta}}-\frac{\overline{\partial \epsilon^{*}\left(\boldsymbol{\theta}_{0}\right)}}{\partial \boldsymbol{\theta}}\right] \\
&=\frac{1}{\sqrt{n}} \sum_{t=p+1}^{n}\left[\lambda\left(F_{\epsilon}\left(\epsilon_{t}\right)\right)-\mathrm{E}\left\{\lambda\left(F_{\epsilon}\left(\epsilon_{t}\right)\right)\right\}\right]\left[\frac{\partial \epsilon_{t}^{*}\left(\boldsymbol{\theta}_{0}\right)}{\partial \boldsymbol{\theta}}-\mathrm{E}\left\{\frac{\partial \epsilon_{t}^{*}\left(\boldsymbol{\theta}_{0}\right)}{\partial \boldsymbol{\theta}}\right\}\right] \\
&-\left(\frac{1}{\sqrt{n}} \sum_{t=p+1}^{n}\left[\lambda\left(F_{\epsilon}\left(\epsilon_{t}\right)\right)-\mathrm{E}\left\{\lambda\left(F_{\epsilon}\left(\epsilon_{t}\right)\right)\right\}\right]\right)\left(\frac{\overline{\partial \epsilon^{*}\left(\boldsymbol{\theta}_{0}\right)}}{\partial \boldsymbol{\theta}}-\mathrm{E}\left\{\frac{\partial \epsilon_{t}^{*}\left(\boldsymbol{\theta}_{0}\right)}{\partial \boldsymbol{\theta}}\right\}\right)
\end{aligned}
$$

converges in distribution to $\mathbf{N}$. By the central limit theorem, $n^{-1 / 2} \sum_{t=p+1}^{n}\left[\lambda\left(F_{\epsilon}\left(\epsilon_{t}\right)\right)-\mathrm{E}\left\{\lambda\left(F_{\epsilon}\left(\epsilon_{t}\right)\right)\right\}\right] \stackrel{d}{\rightarrow}$ $\mathrm{N}(0, \tilde{J})$ and, since $\left\{\partial \epsilon_{t}^{*}\left(\boldsymbol{\theta}_{0}\right) / \partial \boldsymbol{\theta}\right\}$ is stationary ergodic with $\mathrm{E}\left|\partial \epsilon_{t}^{*}\left(\boldsymbol{\theta}_{0}\right) / \partial \theta_{i}\right|<\infty \forall i$ (Francq and Zakoïan, 2007), 
$\overline{\partial \epsilon^{*}\left(\boldsymbol{\theta}_{0}\right) / \partial \boldsymbol{\theta}} \stackrel{P}{\rightarrow} \mathrm{E}\left\{\partial \epsilon_{t}^{*}\left(\boldsymbol{\theta}_{0}\right) / \partial \boldsymbol{\theta}\right\}$. Hence, (5.1) equals

$$
\frac{1}{\sqrt{n}} \sum_{t=p+1}^{n}\left[\lambda\left(F_{\epsilon}\left(\epsilon_{t}\right)\right)-\mathrm{E}\left\{\lambda\left(F_{\epsilon}\left(\epsilon_{t}\right)\right)\right\}\right]\left[\frac{\partial \epsilon_{t}^{*}\left(\boldsymbol{\theta}_{0}\right)}{\partial \boldsymbol{\theta}}-\mathrm{E}\left\{\frac{\partial \epsilon_{t}^{*}\left(\boldsymbol{\theta}_{0}\right)}{\partial \boldsymbol{\theta}}\right\}\right]+o_{p}(1)
$$

Equation (5.2) converges in distribution to $\mathbf{N}$ by the central limit theorem for martingale differences (Billingsley, 1961).

Lemma 5.2. For any $T \in(0, \infty)$, as $n \rightarrow \infty$,

$$
\sup _{\mathbf{u} \in \Lambda,\|\mathbf{u}\| \leq T}\left|\frac{1}{\sqrt{n}} \sum_{t=p+1}^{n} \mathbf{u}^{\prime}\left[\lambda\left(\frac{R_{t}\left(\boldsymbol{\theta}_{0}+n^{-1 / 2} \mathbf{u}\right)}{n-p+1}\right)-\lambda\left(F_{\epsilon}\left(\epsilon_{t}\right)\right)\right]\left[\frac{\partial \epsilon_{t}\left(\boldsymbol{\theta}_{0}\right)}{\partial \boldsymbol{\theta}}-\frac{\overline{\partial \epsilon\left(\boldsymbol{\theta}_{0}\right)}}{\partial \boldsymbol{\theta}}\right]-\tilde{K} \mathbf{u}^{\prime} \boldsymbol{\Gamma} \mathbf{u}\right|
$$

is $o_{p}(1)$. (Recall that $\Lambda=\Lambda_{1} \times \cdots \times \Lambda_{p+q}$, with $\Lambda_{i}=\mathbb{R}$ if $\theta_{0 i}>0$ and $\Lambda_{i}=[0, \infty)$ if $\theta_{0 i}=0$.)

Proof. If $R_{t, n}(\mathbf{u}):=R_{t}\left(\boldsymbol{\theta}_{0}+n^{-1 / 2} \mathbf{u}\right)$ and $\mathbf{V}_{t, n}^{*}:=\partial \epsilon_{t}\left(\boldsymbol{\theta}_{0}\right) / \partial \boldsymbol{\theta}-\overline{\partial \epsilon\left(\boldsymbol{\theta}_{0}\right) / \partial \boldsymbol{\theta}}$, then (5.3) can be expressed as

$$
\sup _{\mathbf{u} \in \Lambda,\|\mathbf{u}\| \leq T}\left|\frac{1}{\sqrt{n}} \sum_{t=p+1}^{n} \mathbf{u}^{\prime}\left[\lambda\left(\frac{R_{t, n}(\mathbf{u})}{n-p+1}\right)-\lambda\left(F_{\epsilon}\left(\epsilon_{t}\right)\right)\right] \mathbf{V}_{t, n}^{*}-\tilde{K} \mathbf{u}^{\prime} \mathbf{\Gamma u}\right| .
$$

Because the weight function $\lambda$ is left-continuous and $\tilde{K}=\int_{-\infty}^{\infty} f_{\epsilon}(x) d \lambda\left(F_{\epsilon}(x)\right)=\int_{0}^{1} f_{\epsilon}\left(F_{\epsilon}^{-1}(y)\right) d \lambda(y),(5.3)$ equals

$$
\sup _{\mathbf{u} \in \Lambda,\|\mathbf{u}\| \leq T}\left|\int_{0}^{1}\left(\frac{1}{\sqrt{n}} \sum_{t=p+1}^{n} \mathbf{u}^{\prime}\left[I\left\{\frac{R_{t, n}(\mathbf{u})}{n-p+1} \leq y\right\}-I\left\{F_{\epsilon}\left(\epsilon_{t}\right) \leq y\right\}\right] \mathbf{V}_{t, n}^{*}+f_{\epsilon}\left(F_{\epsilon}^{-1}(y)\right) \mathbf{u}^{\prime} \mathbf{\Gamma u}\right) d \lambda(y)\right|,
$$

which is bounded above by

$$
\begin{aligned}
& \sup _{\mathbf{u} \in \Lambda,\|\mathbf{u}\| \leq T, y \in(0,1)}\left|\frac{1}{\sqrt{n}} \sum_{t=p+1}^{n} \mathbf{u}^{\prime}\left[I\left\{\frac{R_{t, n}(\mathbf{u})}{n-p+1} \leq y\right\}-I\left\{F_{\epsilon}\left(\epsilon_{t}\right) \leq y\right\}\right] \mathbf{V}_{t, n}^{*}+f_{\epsilon}\left(F_{\epsilon}^{-1}(y)\right) \mathbf{u}^{\prime} \boldsymbol{\Gamma} \mathbf{u}\right| \\
& \quad \times \quad[\lambda(1)-\lambda(0)] .
\end{aligned}
$$

Equation (5.4) is $o_{p}(1)$; a related result is obtained in Andrews (2008, proof of Lemma 5.5) in the case of rank-based estimation for autoregressive-moving average models and a similar proof can be used here, so we omit the details. Since $\lambda$ is bounded, the proof of this lemma is complete.

Next consider the mixed partial derivatives of $\left\{\epsilon_{t}(\boldsymbol{\theta})\right\}_{t=1}^{n}$ :

$$
\frac{\partial^{2} \epsilon_{t}(\boldsymbol{\theta})}{\partial \theta_{i} \partial \theta_{j}}=\frac{1}{\tilde{\sigma}_{t}^{4}(\boldsymbol{\theta})}\left(\frac{\partial \tilde{\sigma}_{t}^{2}(\boldsymbol{\theta})}{\partial \theta_{i}} \frac{\partial \tilde{\sigma}_{t}^{2}(\boldsymbol{\theta})}{\partial \theta_{j}}-\tilde{\sigma}_{t}^{2}(\boldsymbol{\theta}) \frac{\partial^{2} \tilde{\sigma}_{t}^{2}(\boldsymbol{\theta})}{\partial \theta_{i} \partial \theta_{j}}\right), \quad i, j \in\{1, \ldots, p+q\},
$$


with

$$
\frac{\partial^{2} \tilde{\sigma}_{t}^{2}(\boldsymbol{\theta})}{\partial \theta_{i} \partial \theta_{j}}= \begin{cases}0, & i, j \in\{1, \ldots, p\}, \\ \frac{\partial \tilde{\sigma}_{t+p-j}^{2}(\boldsymbol{\theta})}{\partial \theta_{i}}+\sum_{k=1}^{q} \theta_{p+k} \frac{\partial^{2} \tilde{\sigma}_{t-k}^{2}(\boldsymbol{\theta})}{\partial \theta_{i} \partial \theta_{j}}, & i \in\{1, \ldots, p\}, j \in\{p+1, \ldots, p+q\} \\ \frac{\partial \tilde{\sigma}_{t+p-j}^{2}(\boldsymbol{\theta})}{\partial \theta_{i}}+\frac{\partial \tilde{\sigma}_{t+p-i}^{2}(\boldsymbol{\theta})}{\partial \theta_{j}}+\sum_{k=1}^{q} \theta_{p+k} \frac{\partial^{2} \tilde{\sigma}_{t-k}^{2}(\boldsymbol{\theta})}{\partial \theta_{i} \partial \theta_{j}}, & i, j \in\{p+1, \ldots, p+q\}\end{cases}
$$

for $t \in\{p+1, \ldots, n\}$, and $\partial^{2} \tilde{\sigma}_{t}^{2}(\boldsymbol{\theta}) /\left(\partial \theta_{i} \partial \theta_{j}\right)=0$ for $t \leq p$. Let

$$
\overline{\frac{\partial^{2} \epsilon(\boldsymbol{\theta})}{\partial \boldsymbol{\theta} \partial \boldsymbol{\theta}^{\prime}}}=\frac{1}{n-p} \sum_{t=p+1}^{n} \frac{\partial^{2} \epsilon_{t}(\boldsymbol{\theta})}{\partial \boldsymbol{\theta} \partial \boldsymbol{\theta}^{\prime}}
$$

Lemma 5.3. For any $T \in(0, \infty)$, as $n \rightarrow \infty$,

$$
\sup _{\mathbf{u}, \mathbf{v} \in \Lambda,\|\mathbf{u}\|,\|\mathbf{v}\| \leq T}\left|\frac{1}{n} \sum_{t=p+1}^{n} \mathbf{u}^{\prime} \lambda\left(\frac{R_{t}\left(\boldsymbol{\theta}_{0}+n^{-1 / 2} \mathbf{u}\right)}{n-p+1}\right)\left[\frac{\partial^{2} \epsilon_{t}\left(\boldsymbol{\theta}_{0}+n^{-1 / 2} \mathbf{v}\right)}{\partial \boldsymbol{\theta} \partial \boldsymbol{\theta}^{\prime}}-\frac{\overline{\partial^{2} \epsilon\left(\boldsymbol{\theta}_{0}+n^{-1 / 2} \mathbf{v}\right)}}{\partial \boldsymbol{\theta} \partial \boldsymbol{\theta}^{\prime}}\right] \mathbf{u}\right| \stackrel{P}{\rightarrow} 0 .
$$

Proof. For any $i, j \in\{1, \ldots, p+q\}$, the sequence $\left\{\partial^{2} \epsilon_{t}^{*}\left(\boldsymbol{\theta}_{0}\right) /\left(\partial \theta_{i} \partial \theta_{j}\right)\right\}$ of mixed partial derivatives of $\left\{\epsilon_{t}^{*}(\boldsymbol{\theta})\right\}$ at $\boldsymbol{\theta}=\boldsymbol{\theta}_{0}$ is stationary, ergodic with $\mathrm{E}\left|\partial^{2} \epsilon_{t}^{*}\left(\boldsymbol{\theta}_{0}\right) /\left(\partial \theta_{i} \partial \theta_{j}\right)\right|<\infty$ and $n^{-1} \sum_{t=p+1}^{n} \mid \partial^{2} \epsilon_{t}\left(\boldsymbol{\theta}_{0}\right) /\left(\partial \theta_{i} \partial \theta_{j}\right)-$ $\partial^{2} \epsilon_{t}^{*}\left(\boldsymbol{\theta}_{0}\right) /\left(\partial \theta_{i} \partial \theta_{j}\right) \mid \stackrel{P}{\rightarrow} 0$ (Francq and Zakoїan, 2007). Following Lemma 5.1, it can therefore be shown that

$$
\sup _{\mathbf{u} \in \Lambda,\|\mathbf{u}\| \leq T}\left|\frac{1}{n} \sum_{t=p+1}^{n} \mathbf{u}^{\prime} \lambda\left(F_{\epsilon}\left(\epsilon_{t}\right)\right)\left[\frac{\partial^{2} \epsilon_{t}\left(\boldsymbol{\theta}_{0}\right)}{\partial \boldsymbol{\theta} \partial \boldsymbol{\theta}^{\prime}}-\frac{\overline{\partial^{2} \epsilon\left(\boldsymbol{\theta}_{0}\right)}}{\partial \boldsymbol{\theta} \partial \boldsymbol{\theta}^{\prime}}\right] \mathbf{u}\right| \stackrel{P}{\rightarrow} 0
$$

and following Lemma 5.2,

$$
\sup _{\mathbf{u} \in \Lambda,\|\mathbf{u}\| \leq T}\left|\frac{1}{n} \sum_{t=p+1}^{n} \mathbf{u}^{\prime}\left[\lambda\left(\frac{R_{t}\left(\boldsymbol{\theta}_{0}+n^{-1 / 2} \mathbf{u}\right)}{n-p+1}\right)-\lambda\left(F_{\epsilon}\left(\epsilon_{t}\right)\right)\right]\left[\frac{\partial^{2} \epsilon_{t}\left(\boldsymbol{\theta}_{0}\right)}{\partial \boldsymbol{\theta} \partial \boldsymbol{\theta}^{\prime}}-\frac{\overline{\partial^{2} \epsilon\left(\boldsymbol{\theta}_{0}\right)}}{\partial \boldsymbol{\theta} \partial \boldsymbol{\theta}^{\prime}}\right] \mathbf{u}\right| \stackrel{P}{\rightarrow} 0
$$

Since

$$
\sup _{\mathbf{v} \in \Lambda,\|\mathbf{v}\| \leq T} \frac{1}{n} \sum_{t=p+1}^{n}\left|\frac{\partial^{2} \epsilon_{t}\left(\boldsymbol{\theta}_{0}+n^{-1 / 2} \mathbf{v}\right)}{\partial \theta_{i} \partial \theta_{j}}-\frac{\partial^{2} \epsilon_{t}\left(\boldsymbol{\theta}_{0}\right)}{\partial \theta_{i} \partial \theta_{j}}\right| \stackrel{P}{\rightarrow} 0 \quad \forall i, j \in\{1, \ldots, p+q\}
$$

(see Francq and Zakoïan, 2007, proof of Lemma 11), the proof is complete.

Now, for $\mathbf{u} \in \Lambda$ and $\delta_{1}, \delta_{2} \in[0,1]$, let

$$
\begin{aligned}
& \tilde{U}_{n}\left(\mathbf{u}, \delta_{1}, \delta_{2}\right)=\sum_{t=p+1}^{n} \lambda\left(\frac{R_{t}\left(\boldsymbol{\theta}_{0}+n^{-1 / 2} \delta_{1} \mathbf{u}\right)}{n-p+1}\right)\left[\epsilon_{t}\left(\boldsymbol{\theta}_{0}+\frac{\delta_{2} \mathbf{u}}{\sqrt{n}}\right)-\overline{\epsilon\left(\boldsymbol{\theta}_{0}+\frac{\delta_{2} \mathbf{u}}{\sqrt{n}}\right)}\right] \\
& -\sum_{t=p+1}^{n} \lambda\left(\frac{R_{t}\left(\boldsymbol{\theta}_{0}+n^{-1 / 2} \delta_{1} \mathbf{u}\right)}{n-p+1}\right)\left[\epsilon_{t}\left(\boldsymbol{\theta}_{0}+\frac{\delta_{1} \mathbf{u}}{\sqrt{n}}\right)-\overline{\epsilon\left(\boldsymbol{\theta}_{0}+\frac{\delta_{1} \mathbf{u}}{\sqrt{n}}\right)}\right]
\end{aligned}
$$


and

$$
\begin{aligned}
\tilde{V}_{n}\left(\mathbf{u}, \delta_{1}, \delta_{2}\right)= & \sum_{t=p+1}^{n} \lambda\left(\frac{R_{t}\left(\boldsymbol{\theta}_{0}+n^{-1 / 2} \delta_{2} \mathbf{u}\right)}{n-p+1}\right)\left[\epsilon_{t}\left(\boldsymbol{\theta}_{0}+\frac{\delta_{2} \mathbf{u}}{\sqrt{n}}\right)-\overline{\epsilon\left(\boldsymbol{\theta}_{0}+\frac{\delta_{2} \mathbf{u}}{\sqrt{n}}\right)}\right] \\
& -\sum_{t=p+1}^{n} \lambda\left(\frac{R_{t}\left(\boldsymbol{\theta}_{0}+n^{-1 / 2} \delta_{2} \mathbf{u}\right)}{n-p+1}\right)\left[\epsilon_{t}\left(\boldsymbol{\theta}_{0}+\frac{\delta_{1} \mathbf{u}}{\sqrt{n}}\right)-\overline{\left(\boldsymbol{\theta}_{0}+\frac{\delta_{1} \mathbf{u}}{\sqrt{n}}\right)}\right] .
\end{aligned}
$$

Using Taylor series expansions,

$$
\begin{aligned}
\tilde{U}_{n}\left(\mathbf{u}, \delta_{1}, \delta_{2}\right) & \sum_{t=p+1}^{n} \lambda\left(\frac{R_{t}\left(\boldsymbol{\theta}_{0}+n^{-1 / 2} \delta_{1} \mathbf{u}\right)}{n-p+1}\right)\left[\epsilon_{t}\left(\boldsymbol{\theta}_{0}+\frac{\delta_{2} \mathbf{u}}{\sqrt{n}}\right)-\overline{\left(\boldsymbol{\theta}_{0}+\frac{\delta_{2} \mathbf{u}}{\sqrt{n}}\right)}-\epsilon_{t}\left(\boldsymbol{\theta}_{0}\right)+\overline{\epsilon\left(\boldsymbol{\theta}_{0}\right)}\right] \\
& -\sum_{t=p+1}^{n} \lambda\left(\frac{R_{t}\left(\boldsymbol{\theta}_{0}+n^{-1 / 2} \delta_{1} \mathbf{u}\right)}{n-p+1}\right)\left[\epsilon_{t}\left(\boldsymbol{\theta}_{0}+\frac{\delta_{1} \mathbf{u}}{\sqrt{n}}\right)-\overline{\left(\boldsymbol{\theta}_{0}+\frac{\delta_{1} \mathbf{u}}{\sqrt{n}}\right)}-\epsilon_{t}\left(\boldsymbol{\theta}_{0}\right)+\overline{\epsilon\left(\boldsymbol{\theta}_{0}\right)}\right] \\
= & \frac{\delta_{2}-\delta_{1}}{\sqrt{n}} \sum_{t=p+1}^{n} \mathbf{u}^{\prime} \lambda\left(\frac{R_{t}\left(\boldsymbol{\theta}_{0}+n^{-1 / 2} \delta_{1} \mathbf{u}\right)}{n-p+1}\right)\left[\frac{\partial \epsilon_{t}\left(\boldsymbol{\theta}_{0}\right)}{\partial \boldsymbol{\theta}}-\frac{\overline{\partial \epsilon\left(\boldsymbol{\theta}_{0}\right)}}{\partial \boldsymbol{\theta}}\right] \\
& +\frac{\delta_{2}^{2}}{2 n} \sum_{t=p+1}^{n} \mathbf{u}^{\prime} \lambda\left(\frac{R_{t}\left(\boldsymbol{\theta}_{0}+n^{-1 / 2} \delta_{1} \mathbf{u}\right)}{n-p+1}\right)\left[\frac{\partial^{2} \epsilon_{t}\left(\boldsymbol{\theta}_{n}^{*}\left(\mathbf{u}, \delta_{1}, \delta_{2}\right)\right)}{\partial \boldsymbol{\theta} \partial \boldsymbol{\theta}^{\prime}}-\frac{\overline{\partial^{2} \epsilon\left(\boldsymbol{\theta}_{n}^{*}\left(\mathbf{u}, \delta_{1}, \delta_{2}\right)\right)}}{\partial \boldsymbol{\theta} \partial \boldsymbol{\theta}^{\prime}}\right] \mathbf{u} \\
& -\frac{\delta_{1}^{2}}{2 n} \sum_{t=p+1}^{n} \mathbf{u}^{\prime} \lambda\left(\frac{R_{t}\left(\boldsymbol{\theta}_{0}+n^{-1 / 2} \delta_{1} \mathbf{u}\right)}{n-p+1}\right)\left[\frac{\partial^{2} \epsilon_{t}\left(\boldsymbol{\theta}_{n}^{*}\left(\mathbf{u}, \delta_{1}, \delta_{1}\right)\right)}{\partial \boldsymbol{\theta} \partial \boldsymbol{\theta}^{\prime}}-\frac{\overline{\partial^{2} \epsilon\left(\boldsymbol{\theta}_{n}^{*}\left(\mathbf{u}, \delta_{1}, \delta_{1}\right)\right)}}{\partial \boldsymbol{\theta} \partial \boldsymbol{\theta}^{\prime}}\right] \mathbf{u}
\end{aligned}
$$

and, similarly,

$$
\begin{aligned}
\tilde{V}_{n}\left(\mathbf{u}, \delta_{1}, \delta_{2}\right) \\
=\frac{\delta_{2}-\delta_{1}}{\sqrt{n}} \sum_{t=p+1}^{n} \mathbf{u}^{\prime} \lambda\left(\frac{R_{t}\left(\boldsymbol{\theta}_{0}+n^{-1 / 2} \delta_{2} \mathbf{u}\right)}{n-p+1}\right)\left[\frac{\partial \epsilon_{t}\left(\boldsymbol{\theta}_{0}\right)}{\partial \boldsymbol{\theta}}-\frac{\overline{\partial \epsilon\left(\boldsymbol{\theta}_{0}\right)}}{\partial \boldsymbol{\theta}}\right] \\
+\frac{\delta_{2}^{2}}{2 n} \sum_{t=p+1}^{n} \mathbf{u}^{\prime} \lambda\left(\frac{R_{t}\left(\boldsymbol{\theta}_{0}+n^{-1 / 2} \delta_{2} \mathbf{u}\right)}{n-p+1}\right)\left[\frac{\partial^{2} \epsilon_{t}\left(\boldsymbol{\theta}_{n}^{*}\left(\mathbf{u}, \delta_{2}, \delta_{2}\right)\right)}{\partial \boldsymbol{\theta} \partial \boldsymbol{\theta}^{\prime}}-\frac{\overline{\partial^{2} \epsilon\left(\boldsymbol{\theta}_{n}^{*}\left(\mathbf{u}, \delta_{2}, \delta_{2}\right)\right)}}{\partial \boldsymbol{\theta} \partial \boldsymbol{\theta}^{\prime}}\right] \mathbf{u} \\
-\frac{\delta_{1}^{2}}{2 n} \sum_{t=p+1}^{n} \mathbf{u}^{\prime} \lambda\left(\frac{R_{t}\left(\boldsymbol{\theta}_{0}+n^{-1 / 2} \delta_{2} \mathbf{u}\right)}{n-p+1}\right)\left[\frac{\partial^{2} \epsilon_{t}\left(\boldsymbol{\theta}_{n}^{*}\left(\mathbf{u}, \delta_{2}, \delta_{1}\right)\right)}{\partial \boldsymbol{\theta} \partial \boldsymbol{\theta}^{\prime}}-\frac{\overline{\partial^{2} \epsilon\left(\boldsymbol{\theta}_{n}^{*}\left(\mathbf{u}, \delta_{2}, \delta_{1}\right)\right)}}{\partial \boldsymbol{\theta} \partial \boldsymbol{\theta}^{\prime}}\right] \mathbf{u},
\end{aligned}
$$

where the values of $\boldsymbol{\theta}_{n}^{*}(\mathbf{u}, \cdot, \cdot)$ lie between $\boldsymbol{\theta}_{0}$ and $\boldsymbol{\theta}_{0}+n^{-1 / 2} \mathbf{u}$. The functions $\tilde{U}_{n}$ and $\tilde{V}_{n}$ are used in the proof of Lemma 5.4.

Lemma 5.4. For $\mathbf{u} \in \Lambda$, let $S_{n}(\mathbf{u})=D_{n}\left(\boldsymbol{\theta}_{0}+n^{-1 / 2} \mathbf{u}\right)-D_{n}\left(\boldsymbol{\theta}_{0}\right)$ and $S(\mathbf{u})=\mathbf{u}^{\prime} \mathbf{N}+\tilde{K} \mathbf{u}^{\prime} \mathbf{\Gamma} \mathbf{u} / 2$, where $\mathbf{N} \sim N(\mathbf{0}, \tilde{J} \boldsymbol{\Gamma})$. Then $S_{n}(\cdot) \stackrel{d}{\rightarrow} S(\cdot)$ on $C(\Lambda)$, the space of continuous functions on $\Lambda$ where convergence is equivalent to uniform convergence on every compact subset. 
Proof. Let $\mathbf{u} \in \Lambda$ and suppose $m$ is any positive integer. Since

$$
S_{n}(\mathbf{u})=D_{n}\left(\boldsymbol{\theta}_{0}+n^{-1 / 2} \mathbf{u}\right)-D_{n}\left(\boldsymbol{\theta}_{0}\right)=\sum_{k=1}^{m}\left[D_{n}\left(\boldsymbol{\theta}_{0}+\frac{k \mathbf{u}}{m \sqrt{n}}\right)-D_{n}\left(\boldsymbol{\theta}_{0}+\frac{(k-1) \mathbf{u}}{m \sqrt{n}}\right)\right],
$$

we have

$$
\sum_{k=1}^{m} \tilde{U}_{n}\left(\mathbf{u}, \frac{k-1}{m}, \frac{k}{m}\right) \leq S_{n}(\mathbf{u}) \leq \sum_{k=1}^{m} \tilde{V}_{n}\left(\mathbf{u}, \frac{k-1}{m}, \frac{k}{m}\right)
$$

by Theorem 2.1. Using (5.6), (5.7), and Lemmas 5.1, 5.2, and 5.3,

$$
\left[\begin{array}{c}
\tilde{U}_{n}\left(\mathbf{u}, 0, \frac{1}{m}\right) \\
\vdots \\
\tilde{U}_{n}\left(\mathbf{u}, \frac{m-1}{m}, 1\right) \\
\tilde{V}_{n}\left(\mathbf{u}, 0, \frac{1}{m}\right) \\
\vdots \\
\tilde{V}_{n}\left(\mathbf{u}, \frac{m-1}{m}, 1\right)
\end{array}\right] \stackrel{d}{\rightarrow}\left[\begin{array}{c}
\frac{1}{m} \mathbf{u}^{\prime} \mathbf{N}+\frac{0}{m^{2}} \tilde{K} \mathbf{u}^{\prime} \boldsymbol{\Gamma} \mathbf{u} \\
\vdots \\
\frac{1}{m} \mathbf{u}^{\prime} \mathbf{N}+\frac{m-1}{m^{2}} \tilde{K} \mathbf{u}^{\prime} \boldsymbol{\Gamma} \mathbf{u} \\
\frac{1}{m} \mathbf{u}^{\prime} \mathbf{N}+\frac{1}{m^{2}} \tilde{K} \mathbf{u}^{\prime} \boldsymbol{\Gamma} \mathbf{u} \\
\vdots \\
\frac{1}{m} \mathbf{u}^{\prime} \mathbf{N}+\frac{m}{m^{2}} \tilde{K} \mathbf{u}^{\prime} \boldsymbol{\Gamma} \mathbf{u}
\end{array}\right]
$$

on $\mathbb{R}^{2 m}$. Hence,

$$
\left[\begin{array}{c}
\sum_{k=1}^{m} \tilde{U}_{n}\left(\mathbf{u}, \frac{k-1}{m}, \frac{k}{m}\right) \\
\sum_{k=1}^{m} \tilde{V}_{n}\left(\mathbf{u}, \frac{k-1}{m}, \frac{k}{m}\right)
\end{array}\right] \stackrel{d}{\rightarrow}\left[\begin{array}{c}
\mathbf{u}^{\prime} \mathbf{N}+\frac{m-1}{2 m} \tilde{K} \mathbf{u}^{\prime} \boldsymbol{\Gamma} \mathbf{u} \\
\mathbf{u}^{\prime} \mathbf{N}+\frac{m+1}{2 m} \tilde{K} \mathbf{u}^{\prime} \boldsymbol{\Gamma} \mathbf{u}
\end{array}\right]
$$

on $\mathbb{R}^{2}$. For any $\varepsilon>0$, there exists an integer $m$ large enough so that $\mathbf{u}^{\prime} \mathbf{N}+(m-1) /(2 m) \tilde{K} \mathbf{u}^{\prime} \mathbf{\Gamma} \mathbf{u}$ and $\mathbf{u}^{\prime} \mathbf{N}+(m+1) /(2 m) \tilde{K} \mathbf{u}^{\prime} \mathbf{\Gamma u}$ are both in an $\varepsilon$-neighborhood of $S(\mathbf{u})=\mathbf{u}^{\prime} \mathbf{N}+\tilde{K} \mathbf{u}^{\prime} \mathbf{\Gamma} \mathbf{u} / 2$. Thus, for any $\mathbf{u} \in \Lambda$, $S_{n}(\mathbf{u}) \stackrel{d}{\rightarrow} S(\mathbf{u})$. It can be shown similarly that all finite-dimensional distributions of $S_{n}(\cdot)$ converge to those of $S(\cdot)$.

Using (5.8) and an argument in Andrews (2003, pages 84-86), it can be shown that $\lim _{\delta \rightarrow 0^{+}} \limsup _{n \rightarrow \infty} \mathrm{P}\left(\sup _{\mathbf{u}, \mathbf{v} \in K,\|\mathbf{u}-\mathbf{v}\| \leq \delta}\left|S_{n}(\mathbf{u})-S_{n}(\mathbf{v})\right|>\eta\right)=0$ for any $\eta>0$ and any compact subset $K \subset \Lambda$. Therefore, $S_{n}(\cdot)$ is tight on $C(K)$ for any compact set $K \subset \Lambda$. It follows that $S_{n}(\cdot) \stackrel{d}{\rightarrow} S(\cdot)$ on $C(\Lambda)$ by Theorem 7.1 in Billingsley (1999).

Lemma 5.5. Under the conditions of Theorem 3.2, $\sup _{x \in \mathbb{R}}\left|\hat{f}_{n}(x)-f_{\epsilon}(x)\right| \stackrel{P}{\rightarrow} 0$ as $n \rightarrow \infty$.

Proof. If, for $x \in \mathbb{R}, f_{n}(x):=\left(b_{n} n\right)^{-1} \sum_{t=p+1}^{n} \kappa\left(\left[\epsilon_{t}-x\right] / b_{n}\right)$, then $\sup _{x \in \mathbb{R}}\left|f_{n}(x)-f_{\epsilon}(x)\right| \stackrel{P}{\rightarrow} 0$ by Theorem A in Silverman (1978). Using a proof similar to that of Lemma 16 on page 88 of Andrews (2003), it can be shown that $\sup _{x \in \mathbb{R}}\left|\hat{f}_{n}(x)-f_{n}(x)\right| \stackrel{P}{\rightarrow} 0$, and so this lemma holds. 


\section{References}

Abramson, A. \& I. Cohen (2008) Single-sensor audio source separation using classification and estimation approach and GARCH modeling. IEEE Transactions on Audio, Speech and Language Processing 16, 1528-1540.

Andrews, B. (2008) Rank-based estimation for autoregressive moving average time series models. Journal of Time Series Analysis 29, 51-73.

Andrews, B., R.A. Davis, \& F.J. Breidt (2007) Rank-based estimation for all-pass time series models. Annals of Statistics 35, 844-869.

Andrews, D.W.K. (1999) Estimation when a parameter is on a boundary. Econometrica 67, 1341-1383.

Andrews, M.E. (2003) Parameter Estimation for All-Pass Time Series Models. Ph.D. dissertation, Department of Statistics, Colorado State University, Fort Collins, Colorado.

Berkes, I. \& L. Horváth (2004) The efficiency of the estimators of the parameters in GARCH processes. Annals of Statistics 32, 633-655.

Berkes, I., L. Horváth, \& P. Kokoszka (2003) GARCH processes: structure and estimation. Bernoulli 9, 201-227.

Billingsley, P. (1961) The Lindeberg-Lévy theorem for martingales. Proceedings of the American Mathematical Society 12, 788-792.

Billingsley, P. (1999) Convergence of Probability Measures, 2nd ed. New York: Wiley.

Bollerslev, T. (1986) Generalized autoregressive conditional heteroskedasticity. Journal of Econometrics 31, 307327.

Bollerslev, T. (1987) A conditionally heteroskedastic time series model for speculative prices and rates of return. 
Review of Economics and Statistics 69, 542-547.

Bougerol, P. \& N. Picard (1992) Stationarity of GARCH processes and of some nonnegative time series. Journal of Econometrics 52, 115-127.

Brockwell, P.J. \& R.A. Davis (1991) Time Series: Theory and Methods, 2nd ed. New York: Springer.

Campbell, S.D. \& F.X. Diebold (2005) Weather forecasting for weather derivatives. Journal of the American Statistical Association 100, 6-16.

Chernoff, H. \& I.R. Savage (1958) Asymptotic normality and efficiency of certain nonparametric test statistics. Annals of Mathematical Statistics 29, 972-994.

Davis, R.A., K. Knight, \& J. Liu (1992) M-estimation for autoregressions with infinite variance. Stochastic Processes and their Applications 40, 145-180.

Engle, R.F. (1982) Autoregressive conditional heteroscedasticity with estimates of the variance of United Kingdom inflation. Econometrica 50, 987-1008.

Engle, R.F. \& G. González-Rivera (1991) Semiparametric ARCH models. Journal of Business and Economic Statistics 9, 345-359.

Ewing, B.T., J.B. Kruse, \& M.A. Thompson (2008) Analysis of time-varying turbulence in geographicallydispersed wind energy markets. Energy Sources, Part B: Economics, Planning, and Policy 3, 340-347.

Fan, J. \& Q. Yao (2003) Nonlinear Time Series: Nonparametric and Parametric Methods. New York: Springer.

Francq, C. \& J.M. Zakoïan (2004) Maximum likelihood estimation of pure GARCH and ARMA-GARCH processes. Bernoulli 10, 605-637. 
Francq, C. \& J.M. Zakoïan (2007) Quasi-maximum likelihood estimation in GARCH processes when some coefficients are equal to zero. Stochastic Processes and their Applications 117, 1265-1284.

Francq, C. \& J.M. Zakoïan (2009) Testing the nullity of GARCH coefficients: correction of the standard test and relative efficiency comparisons. Journal of the American Statistical Association 104, 313-324.

Gastwirth, J.L. \& S.S. Wolff (1968) An elementary method for obtaining lower bounds on the asymptotic power of rank tests. Annals of Mathematical Statistics 39, 2128-2130.

Hall, P. \& Q. Yao (2003) Inference in ARCH and GARCH models with heavy-tailed errors. Econometrica 71, $285-317$.

Hettmansperger, T.P. \& J.W. McKean (1998) Robust Nonparametric Statistical Methods. London: Arnold.

Hoti, S., M. McAleer, \& F. Chan (2005) Modelling the spillover effects in the volatility of atmospheric carbon dioxide concentrations. Mathematics and Computers in Simulation 69, 46-56.

Huang, H.-H., Y.-M. Shiu, \& P.-S. Lin (2008) HDD and CDD option pricing with market price of weather risk for Taiwan. Journal of Futures Markets 28, 790-814.

Jaeckel, L.A. (1972) Estimating regression coefficients by minimizing the dispersion of the residuals. Annals of Mathematical Statistics 43, 1449-1458.

Jurečková, J. \& P.K. Sen (1996) Robust Statistical Procedures: Asymptotics and Interrelations. New York: Wiley.

Koul, H.L. (2002) Weighted Empirical Processes in Dynamic Nonlinear Models, 2nd ed. New York: Springer.

Koul, H.L. \& S. Ling (2006) Fitting an error distribution in some heteroscedastic time series models. Annals of Statistics 34, 994-1012. 
Mukherjee, K. (2007) Generalized R-estimators under conditional heteroscedasticity. Journal of Econometrics $141,383-415$.

Mukherjee, K. (2008) M-estimation in GARCH models. Econometric Theory 24, 1530-1553.

Muler, N. \& V.J. Yohai (2008) Robust estimates for GARCH models. Journal of Statistical Planning and Inference 138, 2918-2940.

Nelder, J.A. \& R. Mead (1965) A simplex method for function minimization. Computer Journal 7, 308-313.

Nelson, D. (1990) Stationarity and persistence in the GARCH(1,1) model. Econometric Theory 6, 318-334.

Peng, L. \& Q. Yao (2003) Least absolute deviations estimation for ARCH and GARCH models. Biometrika 90, $967-975$.

Rao, C.R. (1973) Linear Statistical Inference and Its Applications, 2nd ed. New York: Wiley.

Romilly, P. (2005) Time series modelling of global mean temperature for managerial decision-making. Journal of Environmental Management 76, 61-70.

Shephard, N. (1996) Statistical aspects of ARCH and stochastic volatility. In D.R. Cox, D.V. Hinkley, \& O.E. Barndorff-Nielsen (eds.) Time Series Models In Econometrics, Finance, and Other Fields, pp. 1-67. London: Chapman \& Hall.

Silverman, B.W. (1978) Weak and strong uniform consistency of the kernel estimate of a density and its derivatives. Annals of Statistics 6, 177-184.

Silverman, B.W. (1986) Density Estimation for Statistics and Data Analysis. New York: Chapman \& Hall.

Straumann, D. (2005) Estimation in Conditionally Heteroscedastic Time Series Models. Berlin: Springer. 